\title{
One-loop corrections to classical masses of kink families
}

\author{
A. Alonso Izquierdo ${ }^{(a, c)}$, W. García Fuertes ${ }^{(b)}$, M.A. González León $^{(c)}$ \\ and J. Mateos Guilarte ${ }^{(d)}$ \\ (a) DAMPT, Cambridge University, UK \\ (b) Departamento de Física, Universidad de Oviedo, SPAIN \\ (c) Departamento de Matemática Aplicada, Universidad de Salamanca, SPAIN \\ (d) Departamento de Física, Universidad de Salamanca, SPAIN
}

\begin{abstract}
One-loop corrections to kink masses in a family of $(1+1)$-dimensional field theoretical models with two real scalar fields are computed. A generalized DHN formula applicable to potentials with and without reflection is obtained. It is shown how half-bound states arising in the spectrum of the second order fluctuation operator for one-component topological kinks and the vacuum play a central rôle in the computation of the kink Casimir energy. The issue of whether or not the kink degeneracy exhibited by this family of models at the classical level survives one-loop quantum fluctuations is addressed .
\end{abstract}

\section{Introduction}

BPS states arising both in extended supersymmetric gauge theories, [1], and string/M theory, [2], play a crucial rôle in the understanding of dualities between the different regimes of these systems. In this framework, domain walls appear as extended states in N=1 SUSY gluodynamics and the Wess-Zumino model, [3]. It is desirable to compute the one-loop quantum corrections, $\Delta M$, to the masses of these new entities. The huge number of fields involved in these theories, however, renders the task impossible. In a search for inspiration about this problem, study of quantum corrections to the masses of $(1+1)$-dimensional real solitons has been reignited in recent years, both in a supersymmetric and a purely bosonic framework.

This topic was first addressed in the classic papers of Dashen, Hasslacher and Neveu, [4], and Faddeev and Korepin, [5] . The authors treated the purely bosonic $\lambda[\phi]_{2}^{4}$ and sine-Gordon models for a single real scalar field. In the first model, the quantum correction to the kink mass was established to be $\Delta M=\hbar m\left(\frac{1}{2 \sqrt{6}}-\frac{3}{\sqrt{2} \pi}\right)$. The response given in those papers is currently accepted to be correct. In the eighties, the supersymmetric extension of these theories was studied widely in references such as $[6,7,8,9,10]$. The main concerns were the quantum correction, $\Delta M$, to the SUSY kink mass, and whether or not the quantum Bogomolny bound was saturated. By 
the end of the past century consensus about both questions was reached, mainly based on the papers of the Minnesota, Stony Brook-Vienna and M.I.T. groups: [11], [12] and [13]. In [11] using powerful supersymmetric techniques a new anomaly in the central charge was found, following a conjecture in the second paper of [12]. In [12] a profound analysis of the bosonic issue was achieved. These authors carefully distinguished between the outcomes obtained using two different kinds of cut-off regularization procedure. They found that only the regularization method based on a subtle cut-off in the number of fluctuation modes fits in smoothly with supersymmetry, precisely the method that leads to the same result as in the computation performed by DHN/FK for the bosonic fluctuations. We shall refine the mode number regularization procedure in a way suitable to be applied also to potentials with reflection, carefully approaching the problem from the 1D Levinson theorem as developed in Reference [14]. Dirichlet boundary conditions will be imposed to manage a discrete spectrum; a cut-off in the energy will be considered, and a finite number of modes near threshold must be subtracted in such a way that the numbers of modes counted around the kink and the vacuum are identical; after this, the continuous limit is taken.

Graham and Jaffe [13] also obtained this response, using techniques based on continuous phase shift methods. Moreover, they extended the analysis to the trivial sector in order to compute the one-loop correction to the static energy of one (far separated) kink-antikink configuration ${ }^{1}$. Here the authors also notice one important point: half-bound states in the spectrum of the second order fluctuation operator around the static configuration require a sharper treatment. Weak fluctuations around the $\lambda\left(\phi^{4}\right)_{2}$ and sine-Gordon kinks are governed by Schrödinger operators which involve potentials without reflection. Thus, the kink zero-point energy receives a contribution from half-bound states that is exactly cancelled by the subtraction of identical contributions from halfbound states to the vacuum Casimir energy. In general, this is no longer so and extreme care is needed in dealing with the non-pairing of half-bound states to the kink and the vacuum sectors.

In both [15] and [16], generalized zeta function and heat kernel regularization methods have been used to compute quantum corrections to the mass of SUSY (the first paper) and bosonic (the second one) one-component kinks. This latter procedure directly uses information coming from the potential - no need to unveil the spectrum - of the Schödinger operator and one skips the subtleties posed by half- bound states. In fact, the heat kernel high-temperature expansion, see [17], allowed us to express the one-loop correction to the $\lambda\left(\phi^{4}\right)_{2}$-kink mass as an asymptotic series with a relative error of the $0.07 \%$.

The main merit of this approach is the breaking of the number of field components barrier. So far, only kinks with a single non-null component were susceptible of being treated semi-classically; the difficulty lies in the study of the spectrum of $k \times k$ non-diagonal matrices of differential operators. In Reference [18], however, the one-loop correction to the mass of two-component topological kinks in the celebrated MSTB model -[19] - has been given as an asymptotic series, starting from the heat kernel expansion.

In order to be complete we also mention that there are other two interesting regularization methods to calculate corrections to kink masses. First, see [20], a local mode regularization - the cutoff reflecting the spacial variation of the kink - conceptually improves the mode number method and leads to the correct answer. Second, see [21], the dimensional regularization procedure is the appropriate method and has been successfully used when the kink is embedded in a domain wall.

In this paper we shall discuss a model encompassing two real scalar fields proposed by Bazeia

\footnotetext{
${ }^{1}$ The kink-antikink system was first correctly analyzed by Schonfeld [9].
} 
and coworkers, see [22], where the next level of complexity in computing quantum corrections to kink masses arises. The main novelty with respect to the MSTB model is the existence of classically degenerate kink families, see [23] and [24]. The importance of the model lies in the fact that Shifman and Voloshin [23] have shown this system to be the dimensional reduction of a generalized Wess-Zumino model with two chiral super-fields. Thus, in this framework the kink solutions turn into BPS domain walls of a effective supersymmetric theory. An explicit demonstration of the stability of some of these solutions is presented in ref. [25] using techniques of SUSY quantum mechanics. More recently, see [26], it has been shown how to modify the supersymmetric version of this model to make the system compatible with local supersymmetry. The kinks of the $(1+1)$ dimensional model become exact extended solutions of $\mathcal{N}=1(3+1)$-dimensional supergravity with the local superpotential proposed by Eto-Sakai. Study of the effective dynamics arising from quantum fluctuations around these kinks is of great interest because they become exact domain walls (two-branes) in $\mathcal{N}=1$ supergravity.

Whether or not the BPS kinks remain degenerate in mass -or the BPS domain walls in surface tension- after taking into account one-loop quantum corrections is the main concern of this investigation. A clue to answering this question is offered in our paper [27]. The low-energy dynamics of BPS kinks is shown to be determined by geodesic motion in the kink moduli space for a special value of the coupling constant. Bohr-Sommerfeld quantization of this adiabatic evolution amounts to treating the Laplace-Beltrami operator of the metric inherited from the kink kinetic energy as the Hamiltonian. The kink moduli space is the half-plane and the metric is flat: Therefore, the Hamiltonian is the ordinary Laplacian for an appropriate choice of coordinates and it seems that the quantum effects do not lift the kink degeneracy, at least in this quantum adiabatic limit. We shall show, however, that these expectations are not fulfilled at the semi-classical level and repulsive forces between separate lumps arise.

The organization of the paper is as follows: in Section $\S 2$ we introduce the model and briefly describe the structure of the configuration space and the rich variety of kinks. Section $\S 3$ is devoted to computing the quantum correction, $\Delta M$, to the one-component topological kink TK1 by means of a generalized DHN procedure applicable to potentials with and without reflection. The contribution of the half-bound states as a function of the coupling constant is carefully analyzed. In this Section the one-loop corrections to the mass of the TK1 kinks are also estimated, using the zeta function regularization method and the high-temperature expansion. Comparison between the approximate and exact results for $\Delta M(\mathrm{TK} 1)$ serves as an evaluation of the error. Starting from numerical solutions, Section $\S 4$ offers a computation of the semi-classical masses for the whole BPS kink variety at three special values of the coupling constant. If $\sigma=2.5$ and $\sigma=1.5$ we find a rapid convergence of the asymptotic series, although violation of the classical degeneracy is suggested by our results. The anomalous kink degeneracy is analyzed in connection with the existence of BPS link kinks. For $\sigma=2$, the system becomes equivalent to two decoupled $\lambda[\phi]_{2}^{4}$ models and the numerical method provides results in agreement with our previous work in [16]: in this case the kink degeneracy still holds at the one-loop level. We also compute in this Section the one-loop corrections to the mass of the BPS link kinks for the value $\sigma=2$ of the couplingconstant in order to easily show that the kink sum rules hold at the semi-classical level. In Section $\S 5$ the dependence of the one-loop kink mass formula on the parameter $\mathrm{c}$ is studied from a general point of view, relying once again on asymptotic methods (the high-temperature expansion). The model also has non-BPS kinks. Section $\S 6$ attempts to elucidate why non-BPS kinks arise in some regimes of the coupling constant. In particular, study of stability via index theorems shows that 
families of this kind of kinks exist for some critical values of the coupling constant. Finally, an Appendix is offered explaining the generalized DHN formula, derived using the cut-off in the mode number regularization procedure but also valid for potentials with reflection.

\section{The model: classical kink degeneracy}

We shall focus on the (1+1)-dimensional two-component scalar field model introduced in [22]. This system arose as the bosonic sector of a supersymmetric theory with superpotential:

$$
\bar{W}(\vec{\chi})=\lambda\left(\frac{1}{3} \chi_{1}^{3}-a^{2} \chi_{1}\right)+\frac{1}{2} \mu \chi_{1} \chi_{2}^{2}
$$

Here $\vec{\chi}\left(y^{\mu}\right)=\chi_{1}\left(y^{\mu}\right) \vec{e}_{1}+\chi_{2}\left(y^{\mu}\right) \vec{e}_{2}$ is a two-component real- scalar field; $y^{\mu}, \mu=0,1$, are coordinates in the $\mathbb{R}^{1,1}$ space-time, and $\vec{e}_{a}, a=1,2$, form an orthonormal basis in the the $\mathbb{R}^{2}$ internal space : $\vec{e}_{a} \cdot \vec{e}_{b}=\delta_{a b}$. We also choose the metric $g^{\mu \nu}=\operatorname{diag}(1,-1)$ in $\mathbb{R}^{1,1}$ and a system of units where $c=1$ but keep $\hbar$ explicit. The coupling constants thus have the following units: $[\lambda]=[\mu]=M^{-\frac{1}{2}} L^{-\frac{3}{2}}$ and $\left[a^{2}\right]=M L$. The dimension of the scalar field is: $[\vec{\chi}]=M^{\frac{1}{2}} L^{\frac{1}{2}}$.

The dynamics is governed by the action:

$$
\bar{S}[\vec{\chi}]=\int d^{2} y\left[\frac{1}{2} \partial_{\mu} \vec{\chi} \cdot \partial^{\mu} \vec{\chi}-\frac{1}{2} \vec{\nabla} \bar{W} \cdot \vec{\nabla} \bar{W}\right] \quad .
$$

Introducing dimensionless fields, variables and parameters $\vec{\chi}=2 a \vec{\phi}, y^{1}=\frac{x}{a \lambda}, y^{0}=\frac{t}{a \lambda}$, and $\sigma=\frac{\mu}{\lambda}$, we obtain expressions which are simpler to handle.

For static configurations and the dimensionless superpotential $W(\vec{\phi})=2\left(\frac{1}{3} \phi_{1}^{3}-\frac{1}{4} \phi_{1}+\frac{\sigma}{2} \phi_{1} \phi_{2}^{2}\right)$ the energy functional reads:

$$
\begin{aligned}
& \overline{\mathcal{E}}\left[\chi_{1}, \chi_{2}\right]=4 a^{3} \lambda \mathcal{E}\left[\phi_{1}, \phi_{2}\right] \\
& \mathcal{E}[\phi]=\int d x\left[\frac{1}{2}\left(\frac{d \phi_{1}}{d x}\right)^{2}+\frac{1}{2}\left(\frac{d \phi_{2}}{d x}\right)^{2}+\frac{1}{8}\left(4 \phi_{1}^{2}+2 \sigma \phi_{2}^{2}-1\right)^{2}+2 \sigma^{2} \phi_{1}^{2} \phi_{2}^{2}\right]
\end{aligned}
$$

It is a function of the unique classically relevant coupling constant $\sigma$ and also depends on the dimensionless potential term $U\left(\phi_{1}, \phi_{2}\right)=\frac{1}{8}\left(4 \phi_{1}^{2}+2 \sigma \phi_{2}^{2}-1\right)^{2}+2 \sigma^{2} \phi_{1}^{2} \phi_{2}^{2}$.

There are four classical vacuum configurations in this system, organized in two orbits of the internal parity symmetry group $G_{I}=\mathbb{Z}_{2} \times \mathbb{Z}_{2}$ generated by the transformations $\pi_{1}:\left(\phi_{1}, \phi_{2}\right) \rightarrow$ $\left(-\phi_{1}, \phi_{2}\right)$ and $\pi_{2}:\left(\phi_{1}, \phi_{2}\right) \rightarrow\left(\phi_{1},-\phi_{2}\right)$. The vacuum moduli space, however, includes two points if $\sigma>0$ :

$$
\begin{aligned}
& \mathcal{M}=\frac{G_{I}}{H_{I}^{(1)}} \cup \frac{G_{I}}{H_{I}^{(2)}} \quad, \quad \overline{\mathcal{M}}=\frac{\mathcal{M}}{G_{I}}=\text { point } \cup \text { point } \\
& \vec{\phi}_{V_{1}^{ \pm}}(x, t)= \pm \frac{1}{2} \vec{e}_{1} \quad, \quad \vec{\phi}_{V_{2}^{ \pm}}(x, t)= \pm \frac{1}{\sqrt{2 \sigma}} \vec{e}_{2}
\end{aligned}
$$

Here, $H_{I}^{(a)}$ is the sub-group of $G_{I}$ that leaves the $\vec{\phi}_{V_{a}^{ \pm}}$point invariant. If $\sigma<0$, the $\vec{\phi}_{V_{2}^{ \pm}}$is lost and the vacuum moduli space has only one point in this regime. 

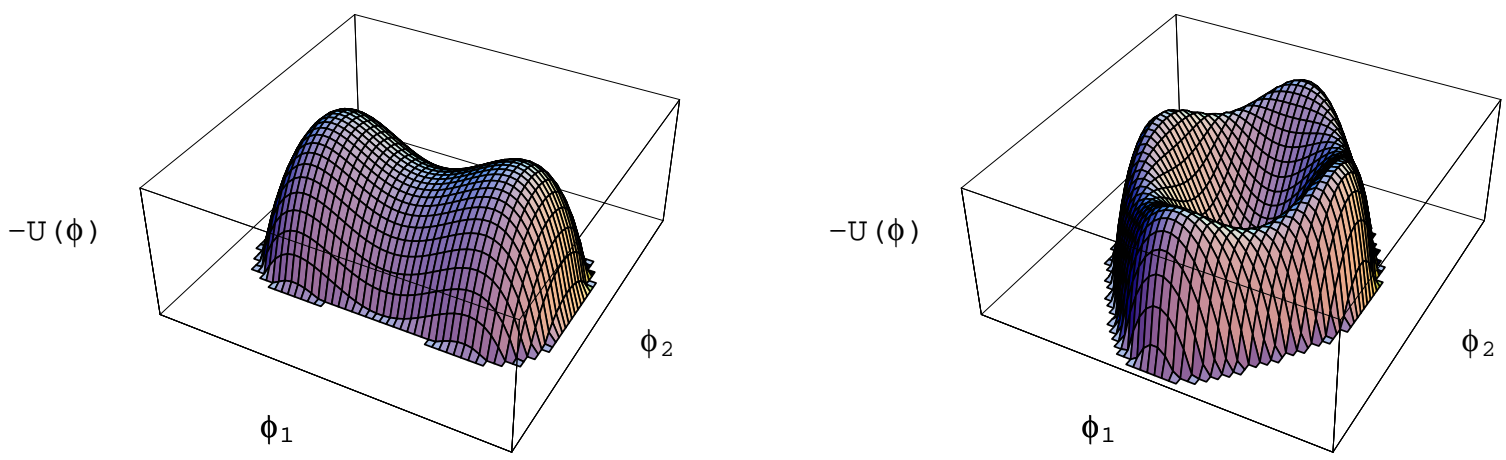

Figure 1: The potential $-U\left(\phi_{1}, \phi_{2}\right)$ for $\sigma<0$ (left) and for $\sigma>0$ (right)

We shall restrict ourselves here to the $\sigma>0$ regime, because a richer manifold of kinks arises in this range. The space of finite energy configurations $\mathcal{C}$ is the union of sixteen disconnected topological sectors in this case. If $a, b=1,2$ we have that:

$$
\mathcal{C}=\sqcup_{a, b} \quad \mathcal{C}_{(a, b)}^{ \pm \pm} \quad \sqcup_{a, b} \quad \mathcal{C}_{(a, b)}^{ \pm \mp}
$$

with

$$
\begin{aligned}
& \mathcal{C}_{(a, b)}^{ \pm \pm}=\left\{\vec{\phi}(x, t) / \vec{\phi}(\infty, t)=\vec{\phi}_{V_{a}^{ \pm}}, \vec{\phi}(-\infty, t)=\vec{\phi}_{V_{b}^{ \pm}}\right\} \\
& \mathcal{C}_{(a, b)}^{ \pm \mp}=\left\{\vec{\phi}(x, t) / \vec{\phi}(\infty, t)=\vec{\phi}_{V_{a}^{ \pm}}, \vec{\phi}(-\infty, t)=\vec{\phi}_{V_{b}^{\mp}}\right\}
\end{aligned}
$$

In the $\mathcal{C}_{(a, a)}^{ \pm \pm}$non-topological sectors a symmetry breaking scenario develops: the original $G_{I}$ symmetry of the action (1) is broken to the $H_{I}^{(a)}$ sub-group by the choice of $\left|\vec{\phi}_{V_{a}^{ \pm}}\right\rangle$as the "zero-particle" state in the $\mathcal{C}_{(a, a)}^{ \pm \pm}$sector.

The small fluctuation solutions $\vec{\eta}_{a}(t, x)=\vec{\phi}_{a}^{ \pm}+\delta \eta(t, x)$ of the field equations

$$
\left(\frac{\partial^{2}}{\partial t^{2}}-\frac{\partial^{2}}{\partial x^{2}}\right) \vec{\phi}=-\vec{\nabla} U
$$

around the vacuum points $V_{1}^{ \pm}$and $V_{2}^{ \pm}$can be respectively expanded in terms of the eigenfunctions of the Hessian operators:

$$
\mathcal{V}_{1}=\left(\begin{array}{cc}
-\frac{d^{2}}{d x^{2}}+4 & 0 \\
0 & -\frac{d^{2}}{d x^{2}}+\sigma^{2}
\end{array}\right) \quad \mathcal{V}_{2}=\left(\begin{array}{cc}
-\frac{d^{2}}{d x^{2}}+2 \sigma & 0 \\
0 & -\frac{d^{2}}{d x^{2}}+2 \sigma
\end{array}\right)
$$

Therefore, the one-particle states have respectively (dimensionless) masses: $m_{1}^{2}\left(V_{1}^{ \pm}\right)=4, m_{2}^{2}\left(V_{1}^{ \pm}\right)=$ $\sigma^{2}$ and $m_{1}^{2}\left(V_{2}^{ \pm}\right)=m_{2}^{2}\left(V_{2}^{ \pm}\right)=2 \sigma$.

The most important feature of this system is that there exists a degenerate in energy family of BPS kinks in the $\mathcal{C}_{(1,1)}^{ \pm \mp}$ topological sectors for any value of $\sigma>0$. BPS kinks are finite energy solutions of the first-order system of differential equations:

$$
\frac{d \phi_{1}}{d x}= \pm \frac{\partial W}{\partial \phi_{1}}= \pm\left(2 \phi_{1}^{2}+\sigma \phi_{2}^{2}-\frac{1}{2}\right) \quad \frac{d \phi_{2}}{d x}= \pm \frac{\partial W}{\partial \phi_{2}}= \pm 2 \sigma \phi_{1} \phi_{2}
$$

In both References [23] and [24] it has been shown that the BPS kinks belonging to the $\mathcal{C}_{(1,1)}^{ \pm \mp}$ topological sectors are in one-to-one correspondence with the curves in the $\mathbb{R}^{2}$ internal space: 
- $\sigma \neq 1$

$$
\phi_{1}^{2}+\frac{\sigma}{2(1-\sigma)} \phi_{2}^{2}=\frac{1}{4}+\frac{c}{2 \sigma}\left|\phi_{2}\right|^{\frac{2}{\sigma}}
$$

where $c \in\left(-\infty, c^{S}\right)$ is an integration constant and there is a critical value $c^{S}=\frac{1}{4} \frac{\sigma}{1-\sigma}(2 \sigma)^{\frac{\sigma+1}{\sigma}}$.

- $\sigma=1$,

$$
\phi_{1}^{2}-\phi_{2}^{2}\left(\frac{c}{2}+\log \left|\phi_{2}\right|\right)=\frac{1}{4}
$$

$c \in\left(-\infty, c^{S}\right), c^{S}=-1+\ln 2$.

One sees in Figure 1 (right) that there are two maxima of $-U\left(\phi_{1}, \phi_{2}\right)$ with the same height. Kink solutions which go from one maximum to the other depend on a parameter $c$ which measures whether the particle moves through the bottom of the valley, or more along the sides on the curve (4). There is a critical value of $c$ where the particle moves as high as possible; increasing $c$ beyond this critical value the particle crosses the mountain and falls off to the other side.

All the BPS kinks belonging to these families have the same (dimensionless) energy: $\mathcal{E}(\mathrm{BPS})=$ $\left|W\left(\vec{\phi}_{V_{1}^{ \pm}}\right)-W\left(\vec{\phi}_{V_{1}^{\mp}}\right)\right|=\frac{1}{3}$. We shall denote these kinks as TK2(c) because from (4) one sees that the two components of the field are non-zero for generic values of $c$; also, all the kink orbits in (4) start and end in vacua belonging to the same $G_{I^{-}}$orbit, and are accordingly classified as "loop" kinks.

Plugging (4) into (3) one reduces the solution of the (3) ODE system to a single quadrature. The explicit integration can be performed analytically only for certain special values of $\sigma$, see References [23], [24].

Exactly for $c=-\infty$, one-component topological kinks - therefore termed as TK1- arise. If $c=-\infty$, for any $\sigma \in[0, \infty)$, we obtain the curve $\phi_{2}=0$ in (4) and find the BPS kinks in the $\mathcal{C}_{(1,1)}^{ \pm \mp}$ sectors of configuration space:

$$
\vec{\phi}_{T K 1}(x)=(-1)^{\alpha} \frac{1}{2} \tanh (x+a) \vec{e}_{1} \quad \alpha \in \mathbb{Z} / \mathbb{Z}_{2}
$$

$a \in \mathbb{R}$ is another integration constant that fixes the center of the kink.

\section{One-loop correction to the mass of one-component topo- logical kinks}

In this Section we shall compute the one-loop quantum correction to the classical mass of TK1 kinks. Because the matrix differential operator governing second-order fluctuations around these kinks is diagonal,

$$
\mathcal{K}=\left(\begin{array}{cc}
-\frac{d^{2}}{d x^{2}}+4-6 \operatorname{sech}^{2} x & 0 \\
0 & -\frac{d^{2}}{d x^{2}}+\sigma^{2}-\sigma(\sigma+1) \operatorname{sech}^{2} x
\end{array}\right)
$$

this task can be performed using both the DHN formula and the asymptotic method. Note also that the one-loop correction to the TK1 kink mass is the sum of two contributions: (1) $\Delta M\left(\mathcal{K}_{11}\right)$ is the one-loop correction due to the the tangent fluctuations to the TK1 kink governed by $\mathcal{K}_{11}$. 
(2) $\Delta M\left(\mathcal{K}_{22}\right)$ collects the contributions to the quantum corrections coming from the orthogonal fluctuations to the TK1 ruled by $\mathcal{K}_{22}$. Therefore, we write

$$
\Delta M(\mathrm{TK} 1)=\Delta M\left(\mathcal{K}_{11}\right)+\Delta M\left(\mathcal{K}_{22}\right)
$$

\subsection{One-loop correction to the TK1 kink mass: DHN formula}

In (5), both $\mathcal{K}_{11}$ and $\mathcal{K}_{22}$ are particular cases of Schrödinger operators of Pösch-Teller type

$$
\Delta=-\frac{d^{2}}{d x^{2}}+c_{0}^{2}-\frac{u_{0}}{\cosh ^{2} x}
$$

whose eigenvalues and eigenfunctions are known [28]. Since $\mathcal{K}_{22}$ is a Schrödinger operator involving potential terms with reflection $(\sigma \notin \mathbb{N})$ and without reflection $(\sigma \in \mathbb{N})$, we use the generalized DHN formula for the $\hbar$-correction to the mass of one-component topological kinks:

$$
\begin{aligned}
\Delta M\left(\mathcal{K}_{a a}\right) & =\frac{\hbar m}{2}\left[\sum_{i=0}^{l-1} \omega_{i}+s_{l} \omega_{l}-\frac{v_{a}}{2}+\frac{1}{\pi} \int_{0}^{\infty} d q \frac{\partial \delta_{a}(q)}{\partial q} \sqrt{q^{2}+v_{a}^{2}}-\frac{\left\langle V_{a a}(x)\right\rangle}{2 \pi}\right]+ \\
& +\hbar m \frac{\left\langle V_{a a}(x)\right\rangle}{8 \pi} \int_{0}^{\infty} \frac{d k}{\sqrt{k^{2}+v_{a}^{2}}} \quad,
\end{aligned}
$$

where

$$
v_{a}^{2}=\left.\frac{\delta^{2} U}{\delta \phi_{a}^{2}}\right|_{\vec{\phi}_{V_{1}^{ \pm}}} \quad ; \quad V_{a a}(x)=v_{a}^{2}-\left.\frac{\delta^{2} U}{\delta \phi_{a}^{2}}\right|_{\vec{\phi}_{T K 1}}
$$

and $\langle\cdot\rangle$ stands for the expectation value: $\left\langle V_{a a}(x)\right\rangle=\int_{-\infty}^{\infty} d x V_{a a}(x)$.

Formula (6) has been derived in the Appendix collecting previous work in this topic. We set as $m=a \lambda$ the parameter with dimension of $L^{-1}$ that will be used to fix the real dimension of each observable in the system. Note that as a consequence of our choice of dimensionless variables there is a global factor of $\sqrt{2}$ in formula (6) with respect to the analogous formula used in [16]. We now pause to explain the origin of the different terms:

1. The first line accounts for the zero-point energy of the quantum kink measured with respect to the zero-point energy of the vacuum. Eigenstates from both the discrete and continuous spectrum of $\mathcal{K}_{a a}$ contribute. The highest bound state contributes with a weight $s_{l}$, which is 1 if $\omega_{l}$ does not coincide with the bottom of the continuous spectrum (threshold). If $\omega_{l}$ is buried at threshold, the corresponding eigenstate is a half-bound state and $s_{l}=\frac{1}{2}$. To deduce formula (6) the density of states in the continuous spectrum is given in terms of the phase shifts $\delta_{a}(q)$ of the scattering waves through the potential: $v_{a}^{2}-V_{a a}(x)$. A cut-off in the number of modes (see Appendix for a complete derivation of formula (6) including the case of potentials with non-zero reflection coefficients) has been used for renormalizing the zero point energy. Note also that the subtraction of $\frac{v_{a}}{2}$ amounts to taking into account the contribution of the half-bound state in the vacuum to the vacuum zero point energy (see [13] and the Appendix).

2. The second line takes into account the contribution of the mass renormalization counter-term to the Casimir energies of both the kink and the vacuum. 


\subsubsection{Tangent fluctuations: $\Delta M\left(\mathcal{K}_{11}\right)$}

$\operatorname{Spec}\left(\mathcal{K}_{11}\right)$ includes two bound states, one half-bound state (in the above notation $l=2$ ), and scattering eigenfunctions characterized by the phase shifts:

$$
\delta_{1}(q)=-2 \arctan \frac{3 q}{2-q^{2}}
$$

Therefore:

$$
\operatorname{Spec}\left(\mathcal{K}_{11}\right)=\left\{\omega_{0}=0\right\} \cup\left\{\omega_{1}=3\right\} \cup\left\{\omega_{2}=4\right\}_{s_{2}=\frac{1}{2}} \cup\left\{q^{2}+4\right\}_{q \in \mathbb{R}} .
$$

Substituting all this information into formula (6) we obtain:

$$
\begin{aligned}
\Delta M\left(\mathcal{K}_{11}^{\mathrm{TK} 1}\right) & =\frac{\sqrt{3} \hbar m}{2}-\frac{\hbar m}{2 \pi} \int_{-\infty}^{\infty} d q \frac{3 \sqrt{q^{2}+4}\left(q^{2}+2\right)}{q^{4}+5 q^{2}+4}+\frac{3 \hbar m}{2 \pi} \int_{-\infty}^{\infty} \frac{d k}{\sqrt{k^{2}+4}}-\frac{\hbar m}{4 \pi} \int_{-\infty}^{\infty} d x 6 \operatorname{sech}^{2} x \\
& =\hbar m\left(\frac{1}{2 \sqrt{3}}-\frac{3}{\pi}\right)
\end{aligned}
$$

Except for a global factor $\sqrt{2}$, the contribution of the tangent fluctuations is the same as the contribution of all the fluctuations to the kink in the $\phi^{4}$ model; note that there are two bound states with eigenvalues of 0 and 3, giving tangent fluctuations to the TK1 kink identical to those arising in the $\lambda\left(\phi^{4}\right)_{2}$ model. The global factor is due to the different choice of the parameters in the potential.

\subsubsection{Orthogonal fluctuations: $\Delta M\left(\mathcal{K}_{22}\right)$}

The scattering in the potential well - $c_{0}^{2}=\sigma^{2}, u_{0}=\sigma(\sigma+1)$ - of $\mathcal{K}_{22}$ is not "reflectionless" if $\sigma$ is not an integer. There are in general even and odd phase shifts

$$
\delta_{2}^{ \pm}(q)=\frac{1}{4} \arctan \left(\frac{\operatorname{Im}(T(q) \pm R(q))}{\operatorname{Re}(T(q) \pm R(q))}\right)
$$

to be read from the transmission and reflection coefficients

$$
T(q)=\frac{\Gamma(\sigma+1-i q) \Gamma(-\sigma-i q)}{\Gamma(1-i q) \Gamma(-i q)} \quad ; \quad R(q)=\frac{\Gamma(\sigma+1-i q) \Gamma(-\sigma-i q) \Gamma(i q)}{\Gamma(1+\sigma) \Gamma(-\sigma) \Gamma(-i q)} \quad .
$$

Recalling that

$$
e^{i 2 \delta_{2}^{ \pm}}=T(q) \pm R(q)
$$

$T(q)$ and $R(q)$ are obtained from the asymptotic behaviour of the scattering eigenstates:

$$
\psi_{q}(x)=N e^{i q x} F_{1}\left[-\sigma, \sigma+1,1-i q, \frac{e^{x}}{e^{x}+e^{-x}}\right] \quad,
$$

where ${ }_{2} F_{1}[a, b, c ; d]$ is the Gauss hypergeometric function .

If $\sigma=l \in \mathbb{N}$ is a natural number, $R(q)=0-\Gamma(-\sigma)$ has a pole in this case-, $\delta_{2}^{+}(q)=\delta_{2}^{-}(q)$, and the total phase shift $\delta_{2}(q)=\delta_{2}^{+}(q)+\delta_{2}^{-}(q)$ is:

$$
\delta_{2}(q)=\frac{1}{2} \arctan \left(\frac{\operatorname{Im} \prod_{n=0}^{l-1}\left(q^{2}-(l-n)^{2}+2 i q(l-n)\right)}{\operatorname{Re} \prod_{n=0}^{l-1}\left(q^{2}-(l-n)^{2}+2 i q(l-n)\right)}\right) .
$$


The number of bound and half-bound states of $\operatorname{Spec}\left(\mathcal{K}_{22}\right)$ is equal to $I[\sigma], I[\sigma]$ being the integer part of $\sigma$. The corresponding eigenvalues are $\omega_{i}=i(2 \sigma-i)$, see [29, 28], whereas the bound state eigenfunctions read:

$$
\psi_{i}(x)=\frac{N}{(\cosh x)^{(\sigma-i)}}{ }^{2} F_{1}\left[-i, 2 \sigma-i+1, \sigma-i+1, \frac{1}{2}(1+\tanh x)\right] \quad, i=0,1, \cdots I[\sigma] \quad .
$$

The half-bound state arises only in the case $\sigma=I[\sigma]=l \in \mathbb{N}$. Some explanation about this issue should be provided: The "first" eigenfunction in the continuous spectrum

$$
\psi_{q=0}(x)=N_{2} F_{1}\left[-\sigma, \sigma+1,1 ; \frac{1}{2}(1+\tanh x)\right]
$$

does not belong to the Hilbert space because $\lim _{x \rightarrow \pm \infty} \psi_{q=0}(x) \cong c_{ \pm} x$, except if $u_{0}=l(l+1)$. In this latter case, $\psi_{i=l}=\psi_{q=0}$,

$$
\lim _{x \rightarrow \pm \infty}{ }_{2} F_{1}\left[-l, l+1,1 ; \frac{1}{2}(1+\tanh x)\right] \cong c_{ \pm}
$$

and hence this is the half-bound state in the spectrum of $\mathcal{K}_{22}$.

In sum, we have that:

$\operatorname{Spec}\left(\mathcal{K}_{22}\right)= \begin{cases}\cup_{i=0,1, \ldots, I \sigma \sigma]}\left\{\omega_{i}=i(2 \sigma-i)\right\} \cup\left\{q^{2}+\sigma^{2}\right\}_{q \in \mathbb{R}^{+}} & \text {if } \quad \sigma \notin \mathbb{N} \\ \cup_{i=0,1, \ldots, \sigma-1}\left\{\omega_{i}=i(2 \sigma-i)\right\} \cup\left\{\omega_{l=\sigma}=\sigma^{2}\right\}_{s_{l=\sigma}=\frac{1}{2}} \cup\left\{q^{2}+\sigma^{2}\right\}_{q \in \mathbb{R}^{+}} & \text {if } \quad \sigma \in \mathbb{N}\end{cases}$

Therefore, in the subspace of $\mathcal{C}_{(1,1)}^{ \pm \mp}$ orthogonal to the TK1 configuration more bound states, depending in number on the value of $\sigma^{2}$, appear as eigen-fluctuations of the Hessian operator. The corresponding eigenvalues are semi-definite positive for any value of sigma. The TK1 kink is thus stable independently of the $\sigma^{2}$ parameter. An important point is that there exists a second zero mode because the first eigenvalue of $\mathcal{K}_{22}$ is zero for all $\sigma^{2}$. This zero mode obeys neutral equilibrium fluctuations in a continuous family of kinks with the same energy as the TK1 kink and second non-zero component, see [24].

Formula (6) demands the computation of the derivative of the phase shift with respect to the momentum $q$, using (7) can be performed explictly:

$$
\begin{aligned}
\frac{\partial \delta_{2}(q)}{\partial q} & =-\frac{i}{2}\left[e^{-i 2 \delta_{2}^{+}} \frac{\partial e^{i 2 \delta_{2}^{+}}}{\partial q}+e^{-i 2 \delta_{2}^{-}} \frac{\partial e^{i 2 \delta_{2}^{-}}}{\partial q}\right]= \\
& =2 \operatorname{Re}[\psi(i q)-\psi(-\sigma+i q)]+\frac{\pi}{2 \sinh ^{2} \pi q \csc 2 \pi \sigma+\tan \pi \sigma}
\end{aligned} .
$$

Plugging all these expressions into (6) we have that:

$$
\begin{aligned}
& \Delta M\left(\mathcal{K}_{22}\right)= \\
& =\frac{\hbar m}{2}\left[\sum_{i=0}^{I[\sigma]} \sqrt{i(2 \sigma-i)}-\frac{\sigma}{2}+\frac{1}{\pi} \int_{0}^{\infty} d q\left(\frac{\partial \delta_{2}(q)}{\partial q} \sqrt{q^{2}+\sigma^{2}}+\frac{\sigma(1+\sigma)}{\sqrt{q^{2}+\sigma^{2}}}\right)-\frac{\sigma(\sigma+1)}{\pi}\right] \quad \text { if } \sigma \notin \mathbb{N} \\
& =\frac{\hbar m}{2}\left[\sum_{i=0}^{\sigma-1} \sqrt{i(2 \sigma-i)}+\frac{1}{\pi} \int_{0}^{\infty} d q\left(\frac{\partial \delta_{2}(q)}{\partial q} \sqrt{q^{2}+\sigma^{2}}+\frac{\sigma(1+\sigma)}{\sqrt{q^{2}+\sigma^{2}}}\right)-\frac{\sigma(\sigma+1)}{\pi}\right] \quad \text { if } \quad \sigma \in \mathbb{N}
\end{aligned}
$$


We are now interested in describing separately the behaviour of the contributions from the discrete spectrum of the operator $\mathcal{K}_{22}$ and from the integral in (10) to $\Delta M\left(\mathcal{K}_{22}\right)$. We define

$$
\Delta M\left(\mathcal{K}_{22}^{(d)}\right)=\sum_{i=0}^{I[\sigma]} \sqrt{i(2 \sigma-i)} \quad, \quad f(q, \sigma)=\left(\frac{\partial \delta_{2}(q)}{\partial q} \sqrt{q^{2}+\sigma^{2}}+\frac{\sigma(1+\sigma)}{\sqrt{q^{2}+\sigma^{2}}}\right)
$$

to emphasize the different contributions.

In $\Delta M\left(\mathcal{K}_{22}^{(d)}\right)$, the contribution of the discrete spectrum of $\mathcal{K}_{22}$ to the one-loop correction of the TK1 kink mass, we notice the following. First, the greater the value of $\sigma$, the greater the number of bound states in $\operatorname{Spec}\left(\mathcal{K}_{22}\right)$ is. There is a jump in $\Delta M\left(\mathcal{K}_{22}^{(d)}\right)$ when $\sigma$ crosses an integer value, see Figure 2. Second, a half-bound state in $\operatorname{Spec}\left(\mathcal{K}_{22}\right)$ arises exactly at the values $\sigma=l \in \mathbb{N}$, which contributes to $\Delta M\left(\mathcal{K}_{22}^{(d)}\right)$ with a $\frac{1}{2}$ weight with respect to the contribution of any other state. One would think from these considerations that $\Delta M\left(\mathcal{K}_{22}\right)$ is a non-continuous function of the coupling constant $\sigma$ because of the jumps in $\Delta M\left(\mathcal{K}_{22}^{(d)}\right)$. On the other hand, we know that the TK1 solution depends smoothly on the value of $\sigma$, such that a smooth response of $\Delta M\left(\mathcal{K}_{22}\right)$ would be expected.
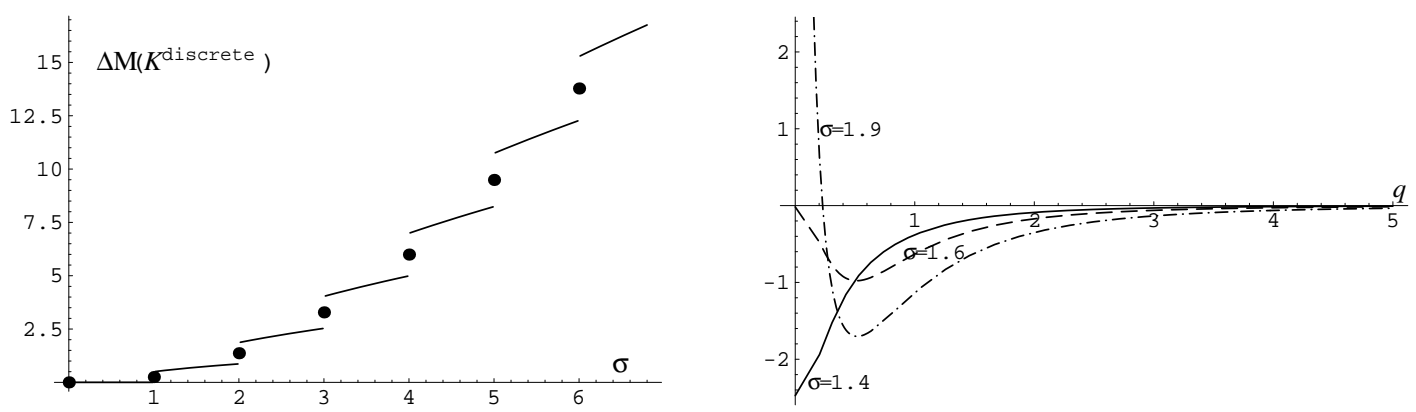

Figure 2: Contribution to the one-loop correction from the discrete spectrum of $\mathcal{K}_{22}$ (left) Behaviour of the integrand $f(q, \sigma)$ in (10) (right)

The analysis of the second contribution is also subtle. The main points are as follows: First, the descriptive behaviour of the integrand $f(q, \sigma)$ depends only on the fractional part of $\sigma$, following the pattern shown in Figure 2 for the range $\sigma \in(1,2)$. Second, asymptotically we have that:

$$
\lim _{q \rightarrow \infty} f(q, \sigma)=0 \quad, \quad \forall \sigma \quad .
$$

Moreover,

$$
\lim _{q \rightarrow 0} f(q, \sigma)=1+\sigma\left(1-2 \gamma_{E}-\psi(-\sigma) \psi(1+\sigma)\right) \quad,
$$

where $\gamma_{E}$ is the Euler Gamma constant, and $\psi(z)$ is the Digamma function. Note that $f(q, \sigma=l)$ has a pole at $q=0$. Because $f(q, \sigma)$ is well-behaved for any other value of $q$ if $\sigma$ is non-integer, the integral in (6) is well defined and a finite answer for $\Delta M\left(\mathcal{K}_{22}\right)$ is obtained, see Figure 2. The integral becomes improper if $\sigma=l \in \mathbb{N}$ : in this case it is only defined by taking the limit $q \rightarrow 0$ appropriately. A miracle happens: the integral of the function $f(q, \sigma)$ produces a jump in this contribution when $\sigma$ crosses an integer that exactly cancels the jump in $\Delta M\left(\mathcal{K}_{22}^{(d)}\right)$ due to the appearance of a new bound or half-bound state!, $\Delta M\left(\mathcal{K}_{22}\right)$ is a continuous function of $\sigma$. 
Nevertheless, the integral cannot be evaluated analytically except for integer values of $\sigma$. We use numerical analysis to compute $\Delta M\left(\mathcal{K}_{22}\right)$ by means of formula (6). The numerical computation confirms that $\Delta M\left(\mathcal{K}_{22}\right)$ is smooth in $\sigma$. The contributions from the discrete and continuous spectrum balance each other to obtain a smooth behaviour in $\sigma$. We stress that this balance is made possible by a proper counting of the half-bound states in both the vacuum and TK1 kink Schrodinger operators.

In the next Table we offer the data on the sum of the tangent and orthogonal contributions to the TK1 quantum mass correction, $\Delta M$ (TK1), for some values in the range $\sigma \in(0.4,3.3)$, which are also depicted in Figure 3.

\begin{tabular}{|c|c|}
\hline$\sigma$ & $\Delta M(\mathrm{TK} 1) / \hbar m$ \\
\hline 0.4 & -0.799335 \\
0.5 & -0.829892 \\
0.6 & -0.860369 \\
0.7 & -0.890955 \\
0.8 & -0.921788 \\
0.9 & -0.952966 \\
0.99 & -0.981384 \\
1.00 & -0.984565 \\
1.01 & -0.98775 \\
1.1 & -1.01664 \\
1.2 & -1.04925 \\
1.3 & -1.08242 \\
\hline
\end{tabular}

\begin{tabular}{|c|c|}
\hline$\sigma$ & $\Delta M(\mathrm{TK} 1) / \hbar m$ \\
\hline 1.4 & -1.11618 \\
1.5 & -1.15057 \\
1.6 & -1.18559 \\
1.7 & -1.22128 \\
1.8 & -1.25765 \\
1.9 & -1.2947 \\
1.99 & -1.32865 \\
2.0 & -1.33251 \\
2.01 & -1.33627 \\
2.1 & -1.37094 \\
2.2 & -1.41013 \\
2.3 & -1.45005 \\
\hline
\end{tabular}

\begin{tabular}{|c|c|}
\hline$\sigma$ & $\Delta M(\mathrm{TK} 1) / \hbar m$ \\
\hline 2.4 & -1.4907 \\
2.5 & -1.53212 \\
2.6 & -1.57427 \\
2.7 & -1.61717 \\
2.8 & -1.65316 \\
2.9 & -1.70527 \\
2.99 & -1.74592 \\
3.0 & -1.72309 \\
3.01 & -1.75503 \\
3.1 & -1.79644 \\
3.2 & -1.84319 \\
3.3 & -1.89071 \\
\hline
\end{tabular}

\subsection{Asymptotic series for the TK1 semi-classical masses}

The evaluation of one-loop corrections to the masses of other (two-component) kinks which are classically degenerate in energy with the TK1 kink is another task to be addressed in this work. For this purpose the DHN formula is of no use, because our knowledge about the spectrum of nondiagonal matrix Schödinger operators is grossly insufficient. Alternatively, we can use a formula, derived in References $[16,18]$, that expresses the one-loop correction to $k$-component kink masses as an asymptotic series. If we denote as $\mathcal{K}$ the Hessian operator around a given loop kink $\mathrm{K}$, the formula for the one-loop correction to the masses of topological kinks derived in Reference [18] reads:

$$
\Delta M(\mathcal{K})=\hbar m\left[\Delta_{0}+D_{n_{0}}\right]\left\{\begin{array}{l}
\Delta_{0}=-\frac{j}{2 \sqrt{\pi}} \\
D_{n_{0}}=-\sum_{a=1}^{2} \sum_{n=2}^{n_{0}-1} \frac{\left[a_{n}\right]_{a a}(\mathcal{K})}{8 \pi} \frac{\gamma\left[n-1, v_{a}^{2}\right]}{v_{a}^{2 n-2}}, \quad n_{0} \in \mathbb{N} .
\end{array}\right.
$$

A lightning summary of the content of formula (12) is as follows: $j=\operatorname{dim} \operatorname{Ker}(\mathcal{K})$ is the number of zero modes in the spectrum of $\mathcal{K} .\left[a_{n}\right]_{a a}(\mathcal{K})$ are the coefficients of the high- temperature expansion of the heat function associated with the heat equation:

$$
\sum_{b=1}^{2}\left(\frac{\partial}{\partial \beta} \delta_{a b}+\mathcal{K}_{a b}\right) F_{b}(\beta, x)=0
$$

$\gamma\left[n-1, v_{a}^{2}\right]$ are incomplete Gamma functions, see [30], and $v_{a}$ are the masses of the fundamental quanta at the point of the vacuum moduli space determined by the loop kink $\mathrm{K}$. The divergent 
terms

$$
\sum_{a=1}^{2} \frac{\left[a_{0}\right]_{a a}(\mathcal{K})}{8 \pi} \frac{\gamma\left[-1, v_{a}^{2}\right]}{v_{a}^{-2}} \quad, \quad \sum_{a=1}^{2} \frac{\left[a_{1}\right]_{a a}(\mathcal{K})}{8 \pi} \gamma\left[0, v_{a}^{2}\right]
$$

lacking in (12) are respectively cancelled by subtracting the zero point vacuum energy, and taking into account the mass renormalization counterterm, see [16] and [18].

Although we have computed $\Delta M(T K 1)$ by means of the DHN formula $(6)$ in the previous Section, we now apply (12) to the TK1 kink in order to test how good the approximation provided by the asymptotic series is with respect to the exact DHN result.

The spectrum of $\mathcal{K}$ comprises two zero modes; henceforth, $j=2$. Moreover, $v_{1}^{2}=4$ and $v_{2}^{2}=\sigma^{2}$ in this case, whereas the Seeley coefficients $\left[a_{n}(\mathcal{K})\right]_{11}$ and $\left[a_{n}(\mathcal{K})\right]_{22}$ are pointed out in References $[16,18]$. The calculations of the partial sums in (12) for several values of $\sigma$ and with $n_{0}=10$ provide the results shown in the next Table:

\begin{tabular}{|c|c|}
\hline$\sigma$ & $\Delta M(\mathrm{TK} 1) / \hbar m$ \\
\hline 0.5 & -0.962386 \\
0.6 & -0.970537 \\
0.7 & -0.981183 \\
0.8 & -0.994487 \\
0.9 & -1.01053 \\
1.0 & -1.0293 \\
\hline
\end{tabular}

\begin{tabular}{|c|c|}
\hline$\sigma$ & $\Delta M(\mathrm{TK} 1) / \hbar m$ \\
\hline 1.1 & -1.05073 \\
1.2 & -1.07468 \\
1.3 & -1.10097 \\
1.4 & -1.12939 \\
1.5 & -1.15971 \\
1.6 & -1.19174 \\
\hline
\end{tabular}

\begin{tabular}{|c|c|}
\hline$\sigma$ & $\Delta M(\mathrm{TK} 1) / \hbar m$ \\
\hline 1.7 & -1.22526 \\
1.8 & -1.2599 \\
1.9 & -1.29571 \\
2.0 & -1.33324 \\
2.1 & -1.37074 \\
2.2 & -1.41007 \\
\hline
\end{tabular}

In Figure 3 the results are plotted as (white) squares and are compared with the data obtained from the DHN procedure, (black) dots. We can check the concordance between the two methods; the relative error for the answer from the asymptotic method is, for instance, $0.79 \%$ for $\sigma=1.5$, $0.055 \%$ for $\sigma=2.0$ and $0.004 \%$ for $\sigma=2.2$. The greater $\sigma$, the more exact the response reached from (12). On the other hand, for the range $\sigma \in(0,1)$ the answer is less precise (for $\sigma=0.9$ the relative error is $6.0 \%$ ). The reason for this becomes clear taking into account that the greater the value of the masses, the smaller the value of $\frac{\gamma\left[n-1, v_{a}^{2}\right]}{v_{a}^{2 n-2}}$, and the more rapidly the series expansion in formula (12) converges.

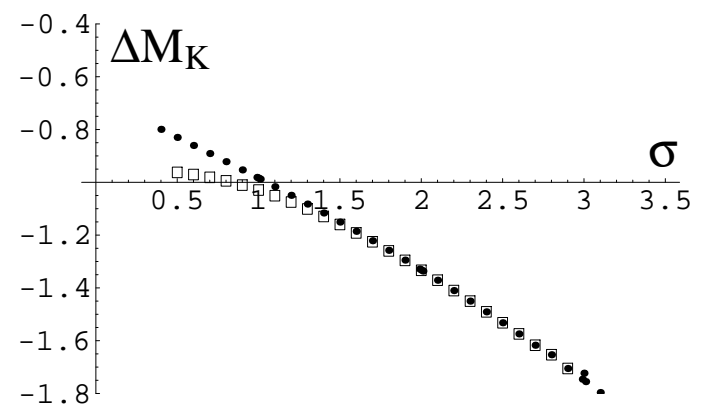

Figure 3: One-loop correction to the one-component topological kink (TK1) mass in units of $\hbar m$. •, DHN formula. . $\square$, asymptotic series.

It is interesting to note that the answer obtained by means of the asymptotic series is a continuous function of $\sigma$. This method, based in the heat kernel expansion and the associated generalized zeta function, leads directly to the correct result, without needing to carefully balance the contributions from the discrete and the continuous parts of the spectrum. The reason is that the Seeley coefficients are defined in terms of the potential (and its derivatives), which encodes all the properties of the spectrum. 


\section{Semi-classical masses of kink families}

So far we have only computed the one-loop correction to the classical mass for the TK1 kinkusing either the (exact) DHN formula (6) or the (approximate) asymptotic expansion (12). The model, however, exhibits a continuous family of kinks which have the same classical energy. Our goal in this Section is to compute the one-loop correction to the kink mass for each member of the family and to analyze the fate of the kink degeneracy in the semi-classical level, using formula (12) with the help of some numerical analysis.

\section{$4.1 \sigma=2$}

In this case, it is possible to find explicit analytical expressions for the solutions corresponding to the kink orbits (4), see [24]:

$$
\vec{\phi}_{T K 2}[x ; a, b]=\frac{(-1)^{\alpha}}{2} \frac{\sinh 2(x+a)}{\cosh 2(x+a)+b} \vec{e}_{1}+\frac{(-1)^{\beta}}{2} \frac{\sqrt{b^{2}-1}}{\cosh 2(x+a)+b} \vec{e}_{2},
$$

where $a \in \mathbb{R}, b=-\frac{c}{\sqrt{c^{2}-16}} \in(1, \infty)$, and $\alpha, \beta \in \mathbb{Z} / \mathbb{Z}_{2}$. The family of Schrödinger operators ruling the small fluctuations in the original fields is in this case

$$
\mathcal{K}(b)=\left(\begin{array}{cc}
-\frac{d^{2}}{d x^{2}}+6 \frac{\sinh ^{2}(2 x)+b^{2}-1}{(\cosh (2 x)+b)^{2}}-2 & 12 \sqrt{b^{2}-1} \frac{\sinh (2 x)}{(\cosh (2 x)+b)^{2}} \\
12 \sqrt{b^{2}-1} \frac{\sinh (2 x)}{(\cosh (2 x)+b)^{2}} & -\frac{d^{2}}{d x^{2}}+6 \frac{\sinh ^{2}(2 x)+b^{2}-1}{(\cosh (2 x)+b)^{2}}-2
\end{array}\right)
$$

The asymptotic method can be applied by substituting the corresponding expressions for this case in formula (12): $j=2, v_{a}^{2}=4, a=1,2$, and the potential terms of the above operator are required in the definitions of the Seeley coefficients $\left[a_{n}\right]_{a a}(\mathcal{K})$. This calculation has been carried out for some kinks of the family (13) with integration constant $c$ in the range $c \in\left[-30, c^{S}\right)$, see Table and Figure 4. In sum, the approximate value of the one-loop quantum mass correction to all these solutions is $-1.33280 \pm 0.00001$ in units of $\hbar m$. Thus, the statement that the degeneracy of the classical mass to the kink family in the case $\sigma=2$ is preserved in the quantum framework is highly accurate.

For this value of $\sigma$ we can easily prove that the statement is completely exact. A rotation of $45^{0}$ in $\mathbb{R}^{2}, \vec{e}_{1}=\frac{1}{\sqrt{2}}\left(\vec{\varepsilon}_{1}+\vec{\varepsilon}_{2}\right), \vec{e}_{2}=\frac{1}{\sqrt{2}}\left(\vec{\varepsilon}_{1}-\vec{\varepsilon}_{2}\right)$, shows that the system is non-coupled. Writing $\vec{\phi}=\psi_{1} \vec{\varepsilon}_{1}+\psi_{2} \vec{\varepsilon}_{2}$, we have that:

$$
T_{\sigma=2}=\frac{1}{2}\left(\frac{d \psi_{1}}{d x}\right)^{2}+\frac{1}{2}\left(\frac{d \psi_{2}}{d x}\right)^{2} \quad, \quad U_{\sigma=2}=4\left(\psi_{1}^{2}-\frac{1}{8}\right)+4\left(\psi_{2}^{2}-\frac{1}{8}\right)
$$

We can write the degenerate kink family in the alternative form:

$$
\vec{\phi}_{T K 2^{*}}\left[x ; a_{1}, a_{2}\right]=\frac{(-1)^{\alpha}}{2 \sqrt{2}} \tanh \left(x+a_{1}\right) \vec{\varepsilon}_{1}+\frac{(-1)^{\beta}}{2 \sqrt{2}} \tanh \left(x+a_{2}\right) \vec{\varepsilon}_{2}
$$

in terms of the new parameters $a_{1}, a_{2} \in(-\infty, \infty)$. Note that the TK1 kinks correspond to $a_{1}=a_{2}$. In these variables the Hessian operator is diagonal for any member of the TK2 family:

$$
\mathcal{K}\left(a_{1}, a_{2}\right)=\left(\begin{array}{cc}
-\frac{d^{2}}{d x^{2}}+4-\frac{6}{\cosh ^{2}\left(x+a_{1}\right)} & 0 \\
0 & -\frac{d^{2}}{d x^{2}}+4-\frac{6}{\cosh ^{2}\left(x+a_{2}\right)}
\end{array}\right)
$$


Therefore, both the spectrum of $\mathcal{K}\left(a_{1}, a_{2}\right)$ and the one-loop correction to the kink masses are independent of $a_{1}, a_{2} \in(-\infty, \infty): \Delta M\left(T K 2^{*}\left[a_{1}, a_{2}\right]\right)=\hbar m\left(\frac{1}{\sqrt{3}}-\frac{6}{\pi}\right) \approx-1.33281$. The kink degeneracy is not broken by quantum fluctuations at the one-loop level; this result would be expected from general arguments because the family of second-order fluctuation operators above is isospectral.

\subsection{Numerical approximation}

The problem that we face is the lack of explicit analytical expressions of the kink solutions for generic values of $\sigma$; only the kink orbits (4) are available. However we can solve the first-order equations (3) by standard numerical methods. We set the "initial" conditions:

$$
\phi_{1}(0)=0 \quad, \quad \frac{\sigma}{2(1-\sigma)} \phi_{2}^{2}(0)-\frac{c}{2 \sigma}\left|\phi_{2}(0)\right|^{\frac{2}{\sigma}}=\frac{1}{4} \quad .
$$

The rationale behind this choice is as follows: (1) for any kink solution, $\phi_{1}(x)$ has always a zero. Translational invariance allows us to set the zero at $x=0$. (2) To ensure that we will find a numerical kink solution, we fix $\phi_{2}(0)$ in such a way that (4) is satisfied for a given value of $\sigma$ and arbitrary choices of $c$.

In this way the numerical method provides us with a succession of points of the kink solution generated by a interpolation polynomial. The kink polynomial is then used to calculate the $\left[a_{n}\right]_{a a}(\mathcal{K})$ coefficients of formula (12) approximately and hence the one-loop correction to the kink mass. A subtle point needs to be clarified: the coefficients are defined in terms of the field solutions and their derivatives. Whereas it is all right to describe the kink solutions approximately by interpolation polynomials, taking "derivatives" of such discrete configurations of points would induce important errors. Fortunately, we can use the first-order equations (3) to express all the field derivatives as polynomials in the fields.

This procedure was carried out for values of $\sigma$ in the $(1.3,3.3)$ range with $\Delta \sigma=0.1$ finding in all cases a similar pattern in the behaviour of the $\Delta M(K(c))$. In the Figure 4 we show the results obtained using this method for several values of $c$ in the range $c \in\left(-30, c^{S}\right)$ in the cases $\sigma=1.5$ and $\sigma=2.5$. A first observation is the perfect agreement reached between the numerical approximation and the exact result of the $\sigma=2$ case. Next, we notice that the greater the value of $\sigma$, the better the convergence of the asymptotic expansion to the exact value for kink solutions with $c$ sufficiently distant from $c^{S}$. We check that the kink degeneracy is always maintained in a wide range of $c$, but starts to fail when $c$ approaches $c^{S}$. 


\begin{tabular}{cc}
$\sigma=1.5$ & \\
\hline$c$ & $\Delta M$ \\
\hline-30 & -1.16009 \\
-27.5 & -1.16017 \\
-25 & -1.16128 \\
-22.5 & -1.16042 \\
-20 & -1.16061 \\
-17.5 & -1.16088 \\
-15 & -1.16128 \\
-12.5 & -1.16193 \\
-10 & -1.16313 \\
-7.5 & -1.16597 \\
-5 & -1.18205 \\
-4.6801886 & -1.24345 \\
-4.68018860186678332 & -1.25103 \\
\hline
\end{tabular}

\begin{tabular}{cc}
$\sigma=2.0$ & \\
\hline$c$ & $\Delta M$ \\
\hline-30 & -1.33281 \\
-27.5 & -1.33281 \\
-25 & -1.33281 \\
-22.5 & -1.33281 \\
-20 & -1.33281 \\
-17.5 & -1.33281 \\
-15 & -1.33281 \\
-12.5 & -1.33281 \\
-10 & -1.33281 \\
-7.5 & -1.33281 \\
-5 & -1.33280 \\
-4.001 & -1.33280 \\
-4.00001 & -1.33280 \\
\hline
\end{tabular}

\begin{tabular}{cc}
$\sigma=2.5$ & \\
\hline$c$ & $\Delta M$ \\
\hline-30 & -1.52784 \\
-27.5 & -1.52782 \\
-25 & -1.52780 \\
-22.5 & -1.52778 \\
-20 & -1.52774 \\
-17.5 & -1.52769 \\
-15 & -1.52760 \\
-12.5 & -1.52744 \\
-10 & -1.52711 \\
-7.5 & -1.52626 \\
-5 & -1.52285 \\
-4 & -1.52168 \\
-3.97 & -1.52915 \\
-3.96594571 & -1.55402 \\
-3.96594570565808127 & -1.56127 \\
\hline
\end{tabular}

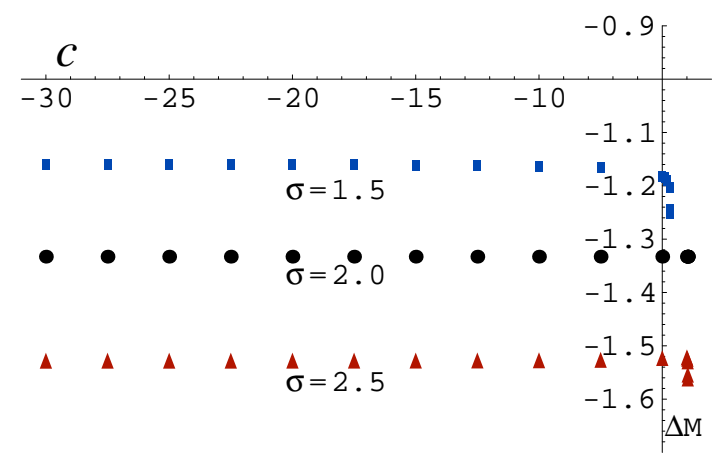

Figure 4: The One-loop Quantum Mass Correction in the cases $\sigma=1.5, \sigma=2.0$ and $\sigma=2.5$.

Thus, the previous Table and Figure suggest the survival of kink degeneracy at the one-loop level up to values of $c$ close to $c^{S}$. A more precise idea about what is going on in the vicinity of $c^{S}$ is given by the following observations:

1) We first notice that the $c=c^{S}$ kink orbits belong to the $\mathcal{C}_{(a, b)}^{ \pm \mp}(a \neq b)$ topological sectors for any value of $\sigma$. Thus, this kind of kink is called a "link" because the associated orbits link different points in the vacuum moduli space. The trajectories of these link kinks enclose the orbits of the kink family studied previously in Section $\S 4$. For $\sigma=2$, these TK2L topological kinks of link type are:

$$
\vec{\phi}_{\mathrm{TK} 2 \mathrm{~L}}(x)=\frac{(-1)^{\alpha}}{4}\left(1-(-1)^{\gamma} \tanh (x+a)\right) \vec{e}_{1}+\frac{(-1)^{\beta}}{4}\left(1+(-1)^{\gamma} \tanh (x+a)\right) \vec{e}_{2}
$$

with $a \in \mathbb{R}, \alpha, \beta, \gamma \in \mathbb{Z} / \mathbb{Z}_{2}$. The link topological kinks for $\sigma=\frac{1}{2}$ are

$$
\vec{\phi}_{\mathrm{TK} 2 \mathrm{~L}}(x)=\frac{(-1)^{\alpha}}{4}\left(1-(-1)^{\beta} \tanh \frac{x+a}{2}\right) \vec{e}_{1}+\frac{(-1)^{\gamma}}{\sqrt{2}} \sqrt{1+(-1)^{\beta} \tanh \frac{x+a}{2}} \vec{e}_{2}
$$

with $\alpha, \beta, \gamma \in \mathbb{Z}$. There are no analytical expressions available for other values of $\sigma$. 
2) Second, we realize that because the existence of these enveloping kinks the energy density of any kink obtained for $c$ near $c^{S}$ is formed by two lumps. We explain the general situation in the $\sigma=\frac{1}{2}$ case. Even though the asymptotic method works poorly for this value of $\sigma$ (with errors higher than a 18\%), the analytical information allows a full description of the situation. The TK2 kink family is given by:

$$
\vec{\phi}_{T K 2}[x ; b]=\frac{(-1)^{\alpha}}{2} \frac{\sinh (x+a)}{\cosh (x+a)+b} \vec{e}_{1}+(-1)^{\beta} \sqrt{\frac{b}{\cosh (x+a)+b}} \vec{e}_{2}
$$

where $a \in \mathbb{R}, b=\frac{1}{\sqrt{1-4 c}} \in(0, \infty)$, and , $\alpha, \beta \in \mathbb{Z} / \mathbb{Z}_{2}$.

In Reference [27], we showed that the splitting into two lumps starts at $c=0$ or $b=1$. In the previous subsection we have observed through numerical computations for any value of $\sigma \neq 2$ that the departure from the TK1 quantum correction also starts at the value of $c$ where the splitting begins. Use of the $c$ parameter is necessary to implement the numerical method, but unsuitable for discussing this phenomenon. Note that $c \in\left(0, c^{s}\right)$ is tantamount to $b \in(1,+\infty)$. $b$ is a measure of the distance between the two lumps, although a highly non-linear one, see [27]. Thus, the breaking of the degeneracy is noticeable when the two lumps are further apart. The induction of this repulsive force by quantum effects can be better understood by looking at the family of the TK2 Hessian operators:

$$
\mathcal{K}(b)=\left(\begin{array}{cc}
-\frac{d^{2}}{d x^{2}}+\frac{3 b}{\cosh x+b}+\frac{6 \sinh ^{2} x}{(\cosh x+b)^{2}}-2 & \frac{6 \sqrt{b} \sinh x}{(\cosh x+b)^{\frac{3}{2}}} \\
\frac{6 \sqrt{b} \sinh x}{(\cosh x+b)^{\frac{3}{2}}} & -\frac{d^{2}}{d x^{2}}+\frac{3}{2} \frac{b}{\cosh x+b}+\frac{3}{4} \frac{\sinh ^{2} x}{(\cosh x+b)^{2}}-\frac{1}{2}
\end{array}\right)
$$

In the Figure 5 we show a plot of the diagonal components of the potential for several values of $c$.

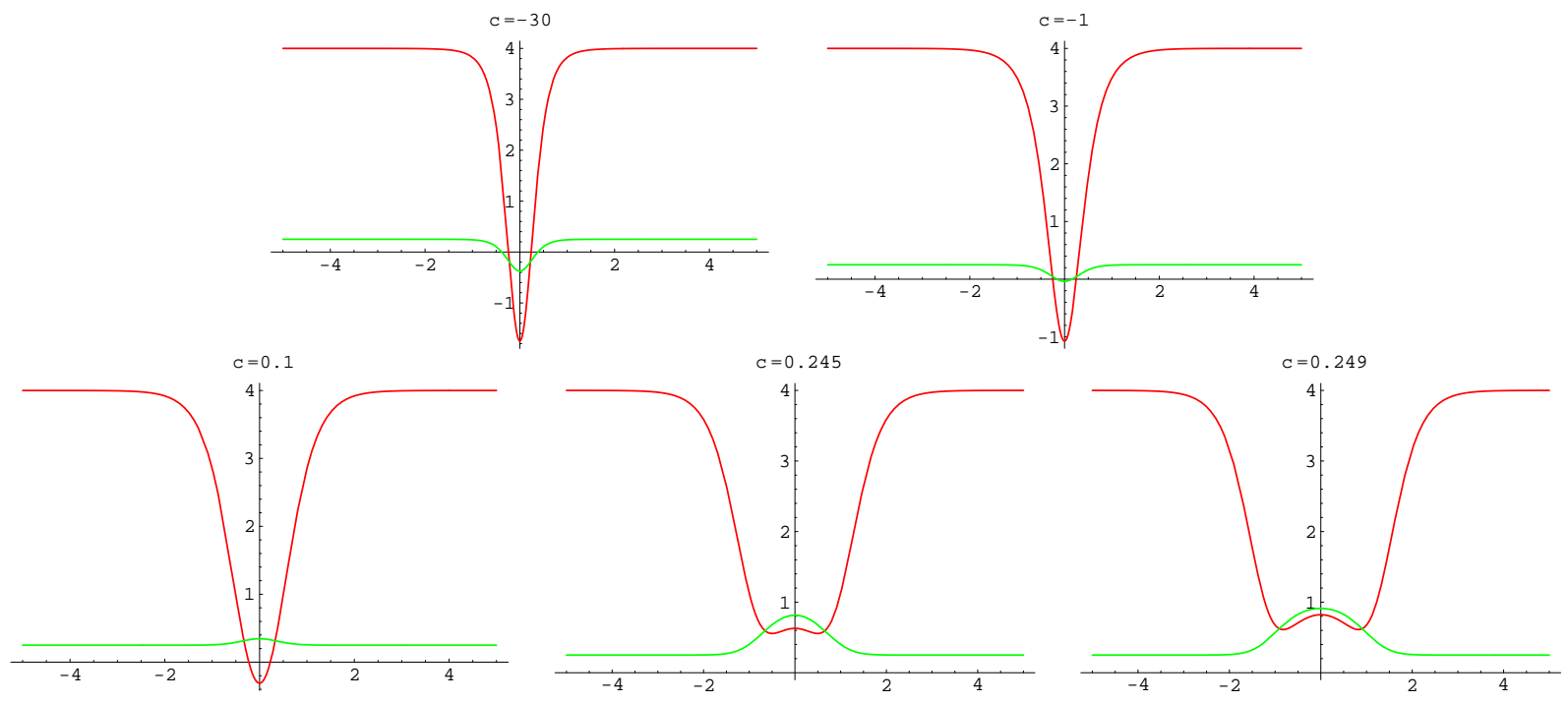

Figure 5: Diagonal components of the potential for $c=-30, c=-1, c=0.1, c=0.245$ and $c=0.249$.

There is no modification of the potential between $c=-30$ and $c=-1$ (the off-diagonal components are odd functions and the area enclosed by them is zero); there must be kink degeneracy in this range of $c$. Starting at $c=0$, the second component turns from potential well to barrier, 
inducing a (still) very weak repulsion. The closer the value of $c$ to $c^{S}=\frac{1}{4}$, the stronger the repulsion coming from the second component, whereas the first component develops a double well, clearly arising from the meiosis into two lumps. Therefore, the spectrum of $\mathcal{K}(b)$ is completely different in the regimes $b \in(0,1)$ and $b \in\left(10^{5}, \infty\right)$, with a transition region in between. We stress again that the picture is similar to this for any $\sigma \neq 2$ : the classical degeneracy starts to fail for the value of $c$ where two peaks in the energy density appear: quantum fluctuations induce repulsive forces between the two basic lumps, which are constituents of a TK2 kink. The data in the Table also show that the rate of change of the quantum correction diminishes when the two lumps are extremely far apart, in a range of $c$ where there are no significant changes in the structure of $\mathcal{K}(c)$.

We stress that the breaking of the kink degeneracy due to quantum effects suggests the possibility of a similar phenomenon between higher dimensional topological defects as vortices and monopoles; it is tantalizing to think that the method here developed could also be applied to study the one-loop effects on the moduli spaces of vortices and monopoles. Nevertheless, one can argue the survival of kink degeneracy to quantum fluctuations in the fully supersymmetric system. The reason is the saturation of the BPS bound at the quantum level in $\mathcal{N}=1$ Wess-Zumino $d=2$ supersymmetric models due to equal anomalies in the energy and the central charge; see Reference [31] for a recent and elegant proof in systems with only one chiral superfield. In our case, two chiral superfields, the anomaly is given - up to some constant- in terms of the Laplacian of the superpotential:

$$
|\Delta W(\vec{\phi}(\infty))-\Delta W(\vec{\phi}(-\infty))|=4+2 \sigma \quad,
$$

a quantity independent of c. Therefore, the c-dependence in the energy coming from bosonic fluctuations must be exactly cancelled by taking into account the fermionic fluctuations with SUSY preserving boundary conditions.

We end this Section by exploring some points about the one-loop correction to the TK2L link kinks. If $\sigma=2$, the TK2L Hessian/Schrodinger operator for $a=0$ is

$$
\mathcal{K}^{L}(\sigma=2)=\left(\begin{array}{cc}
-\frac{d^{2}}{d x^{2}}+4-\frac{3}{\cosh ^{2} x} & \frac{3}{\cosh ^{2} x} \\
{\frac{3}{\cosh ^{2} x}}^{-\frac{d^{2}}{d x^{2}}+4-\frac{3}{\cosh ^{2} x}}
\end{array}\right)
$$

whereas if $\sigma=\frac{1}{2}$, also for $a=0$, we have:

$$
\mathcal{K}^{L}\left(\sigma=\frac{1}{2}\right)=\left(\begin{array}{cc}
-\frac{d^{2}}{d x^{2}}+\frac{5}{2}-\frac{3}{2} \tanh \left(\frac{x}{2}\right)-\frac{3}{2} \frac{1}{\cosh ^{2}\left(\frac{x}{2}\right)} & \frac{3}{2 \sqrt{2}}\left(1-\tanh \left(\frac{x}{2}\right)\right) \sqrt{1+\tanh \left(\frac{x}{2}\right)} \\
\frac{3}{2 \sqrt{2}}\left(1-\tanh \left(\frac{x}{2}\right)\right) \sqrt{1+\tanh \left(\frac{x}{2}\right)} & -\frac{d^{2}}{d x^{2}}+\frac{5}{8}+\frac{3}{8} \tanh \left(\frac{x}{2}\right)-\frac{3}{16} \frac{1}{\cosh ^{2}\left(\frac{x}{2}\right)}
\end{array}\right) .
$$

In this last case, we would need to use the background renormalization method as developed in [16] to compute the one-loop correction, but the task is so involved that we only discuss the $\sigma=2$ case.

It is clear that $\operatorname{Spec} \mathcal{K}^{L}(\sigma=2)=\operatorname{Spec} \mathcal{D}$, where:

$$
\mathcal{D}=\left(\begin{array}{cc}
-\frac{d^{2}}{d x^{2}}+4 & 0 \\
0 & -\frac{d^{2}}{d x^{2}}+4-\frac{6}{\cosh ^{2} x}
\end{array}\right) \quad .
$$

One immediately concludes that

$$
\Delta M(T K 2 L)=\frac{1}{2} \Delta M(T K 1)
$$


i.e. the famous kink sum rule also holds at the one-loop level. The results obtained via the asymptotic expansion agree with this observation. The very accurately calculated degeneracy in the case $\sigma=2$, obtained from numerical plus asymptotic computations even in the vicinity of the limiting value $c^{S}=-4$ (see Figure 4 ), implies that the combination of two identical link kinks (reached when the parameter $c$ goes to $c^{S}$ ) has the same mass quantum correction as the rest of the kinks.

As we have seen the situation is different (and to some extent surprising) for other values of $\sigma$. The singular behaviour of the system for $\sigma=2$ is related to the fact that the metric ruling the kink adiabatic motion is Euclidean if $\sigma=2$, see [27]; consequently, the two lumps move freely with respect to each other.

\section{Classical versus semi-classical kink degeneracy}

In this Section we shall address the subtle question of what happens to the kink degeneracy when quantum effects are taken into account from the analytical - more than the computational- side. Let us denote by

$$
\vec{\phi}^{K(c)}(x ; c)=\bar{\phi}_{1}(x ; c) \vec{e}_{1}+\bar{\phi}_{2}(x ; c) \vec{e}_{2}
$$

the solution of the first-order equations (3) given by the orbit (4). Let us define $\mathcal{K}(c)=\mathcal{V}_{1}-V(c)$ as:

$\mathcal{K}(c)=\left(\begin{array}{cc}-\frac{d^{2}}{d x^{2}}+24 \bar{\phi}_{1}^{2}[x ; c]+4 \sigma(\sigma+1) \bar{\phi}_{2}^{2}[x ; c]-2 & 8 \sigma(\sigma+1) \bar{\phi}_{1} \bar{\phi}_{2}[x ; c] \\ 8 \sigma(\sigma+1) \bar{\phi}_{1} \bar{\phi}_{2}[x ; c] & -\frac{d^{2}}{d x^{2}}+4 \sigma(\sigma+1) \bar{\phi}_{1}^{2}[x ; c]+6 \sigma^{2} \bar{\phi}_{2}^{2}[x ; c]-\sigma\end{array}\right)$

We have thus a family of Schrodinger operators governing the small fluctuations around any kink in the degenerate family.

The one-loop correction to the mass of the $K(c)$ kink is given by the formula (5) of Reference [18]:

$$
\begin{aligned}
\Delta M(K(c)) & =\lim _{s \rightarrow-\frac{1}{2}}\left[\Delta_{1} \varepsilon^{K(c)}(s)+\Delta_{2} \varepsilon^{K(c)}(s)\right] \\
\Delta_{1} \varepsilon^{K(c)}(s) & =\frac{\hbar}{2} \mu^{2 s+1}\left[\zeta_{P \mathcal{K}(c)}(s)-\zeta_{\mathcal{V}_{1}}(s)\right] \\
\Delta_{2} \varepsilon^{K(c)}(s) & =\lim _{L \rightarrow \infty} \frac{\hbar}{2 L} \mu^{2 s+1} \frac{\Gamma(s+1)}{\Gamma(s)} \sum_{a=1}^{2} \zeta_{\left(\mathcal{V}_{1}\right)_{a a}}(s+1) \int_{-\frac{m L}{2}}^{\frac{m L}{2}} d x V_{a a}(x ; c)
\end{aligned}
$$

We recall that $\Delta_{1} \varepsilon^{K(c)}\left(-\frac{1}{2}\right)$ is the kink Casimir energy measured with respect to the vacuum Casimir energy. $\Delta_{2} \varepsilon^{K(c)}\left(-\frac{1}{2}\right)$, however, accounts for the contribution to the kink energy of the mass renormalization counterterm.

The generalized zeta functions of $\mathcal{K}(c)$ (with the zero eigenvalues excluded) and $\mathcal{V}_{1}$ can be written in terms of the heat functions via Mellin transforms:

$$
\zeta_{P \mathcal{K}(c)}(s)=\frac{1}{\Gamma(s)} \int_{0}^{\infty} d \beta \beta^{s-1} \operatorname{Tr} e^{-\beta P \mathcal{K}(c)} \quad, \quad \zeta_{\mathcal{V}_{1}}(s)=\frac{1}{\Gamma(s)} \int_{0}^{\infty} d \beta \beta^{s-1} \operatorname{Tr} e^{-\beta \mathcal{V}_{1}} .
$$

The derivative of $\Delta_{1} \varepsilon^{K(c)}(s)$ with respect to $c$ is obtained immediately,

$$
\frac{\partial}{\partial c} \Delta_{1} \varepsilon^{K(c)}(s)=\frac{\hbar}{2 \Gamma(s)} \mu^{2 s+1} \int_{0}^{\infty} d \beta \beta^{s-1} \operatorname{Tr}\left[\frac{\partial V}{\partial c} e^{-\beta P \mathcal{K}(c)}\right],
$$


whereas we also have:

$$
\frac{\partial}{\partial c} \Delta_{2} \varepsilon^{K(c)}(s)=\lim _{L \rightarrow \infty} \frac{\hbar}{2 L} \mu^{2 s+1} \frac{\Gamma(s+1)}{\Gamma(s)} \sum_{a=1}^{2} \zeta_{\left(\mathcal{V}_{1}\right)_{a a}}(s+1) \int_{-\frac{m L}{2}}^{\frac{m L}{2}} d x \frac{\partial V_{a a}}{\partial c} .
$$

If the sum of these two quantities adds to zero at the $s \rightarrow-\frac{1}{2}$ limit we can be sure that the kink degeneracy also holds up to this order in the $\hbar$-expansion. Unfortunately, an exact computation is beyond our analytical capacities but we can work an asymptotic expansion of this formula along similar lines to those used in Reference [18] and [32]. From the high-temperature expansion of the heat kernel,

$$
K_{\mathcal{K}(c) a b}(x, x ; \beta)=\sum_{d=1}^{2} A_{a d}(x, x ; \beta) \frac{e^{-\beta v_{d}^{2}}}{\sqrt{4 \pi \beta}} \delta_{d b}=\frac{e^{-\beta v_{b}^{2}}}{\sqrt{4 \pi \beta}} \sum_{n=0}^{\infty}\left[a_{n}\right]_{a b}(x, x) \beta^{n}
$$

and bearing in mind that

$$
\begin{aligned}
\operatorname{Tr}\left[\frac{\partial V}{\partial c} e^{-\beta P \mathcal{K}(c)}\right] & =\int_{-\infty}^{\infty} d x \operatorname{tr}\left\langle x\left|\frac{\partial V}{\partial c} e^{-\beta P \mathcal{K}(c)}\right| x\right\rangle \\
& =\int_{-\infty}^{\infty} d x \int_{-\infty}^{\infty} d x^{\prime} \operatorname{tr}\left\langle x\left|\frac{\partial V}{\partial c}\right| x^{\prime}\right\rangle\left\langle x^{\prime}\left|e^{-\beta P \mathcal{K}(c)}\right| x\right\rangle \\
& =\sum_{a, b=1}^{2} \int_{-\infty}^{\infty} d x \frac{\partial V_{a b}(x ; c)}{\partial c} K_{\mathcal{K}(c) b a}(x, x ; \beta)
\end{aligned}
$$

we find

$$
\begin{aligned}
\operatorname{Tr}\left[\frac{\partial V}{\partial c} e^{-\beta \mathcal{K}(c)}\right] & =\frac{1}{\sqrt{4 \pi \beta}} \sum_{n=0}^{\infty} \sum_{a, b=1}^{2} \int_{-\infty}^{\infty} d x\left(\frac{\partial V_{a b}(x)}{\partial c}\left[a_{n}\right]_{b a}(x, x) e^{-\beta v_{a}^{2}}\right) \beta^{n} \\
& =\frac{1}{\sqrt{4 \pi}} \sum_{n=0}^{\infty} \sum_{a=1}^{2}\left[c_{n}\right]_{a} e^{-\beta v_{a}^{2}} \beta^{n-\frac{1}{2}}
\end{aligned}
$$

where

$$
\left[c_{n}\right]_{a}=\sum_{b=1}^{2} \int_{-\infty}^{\infty} d x \frac{\partial V_{a b}(x ; c)}{\partial c}\left[a_{n}\right]_{a b}(x, x)
$$

Therefore,

$$
\frac{\partial}{\partial c} \zeta_{\mathcal{K}(c)}(s)=\frac{1}{\sqrt{4 \pi} \Gamma[s]} \sum_{n=0}^{\infty} \sum_{a=1}^{2}\left[c_{n}\right]_{a} \int_{0}^{1} d \beta e^{-\beta v_{a}^{2}} \beta^{s+n-\frac{1}{2}}+\frac{\partial B_{\mathcal{K}(c)}(s)}{\partial c},
$$

where $B_{\mathcal{K}(c)}(s)$ is a negligible error arising from the truncation of the $\beta$ integration in $\beta=1$. This gives:

$$
\frac{\partial}{\partial c} \Delta_{1} \varepsilon^{K(c)}(s)=\frac{\hbar m}{2} \mu^{2 s+1} \sum_{n=0}^{\infty} \sum_{a=1}^{2} \frac{\left[c_{n}\right]_{a} \gamma\left[s+n+\frac{1}{2}, v_{a}^{2}\right]}{\sqrt{4 \pi} v_{a}^{2\left(s+n+\frac{1}{2}\right)}}+\text { error } \quad .
$$


Also, we have that

$$
\zeta_{\left(\mathcal{V}_{1}\right)_{a a}}(s+1)=\frac{m L}{\Gamma[s+1]} \frac{\gamma\left[s+\frac{1}{2}, v_{a}^{2}\right]}{\sqrt{4 \pi} v_{a}^{2}\left(s+\frac{1}{2}\right)}
$$

and, because $\left[a_{0}\right]_{a b}(y, y)=\delta_{a b}$,

$$
\left[c_{0}\right]_{a}=\sum_{b=1}^{2} \int_{-\infty}^{\infty} d y \frac{\partial V_{a b}}{\partial c}
$$

Thus,

$$
\frac{\partial}{\partial c} \Delta_{2} \varepsilon^{K(c)}(s)=-\frac{\hbar m}{2} \mu^{2 s+1} \sum_{a=1}^{2} \frac{\gamma\left[s+\frac{1}{2}, v_{a}^{2}\right]}{\sqrt{4 \pi} v_{a}^{2}\left(s+\frac{1}{2}\right)}\left[c_{0}\right]_{a}
$$

and finally we obtain:

$$
\frac{\partial}{\partial c}\left[\Delta_{1} \varepsilon^{K(c)}(s)+\Delta_{2} \varepsilon^{K(c)}(s)\right]=\frac{\hbar m}{2} \mu^{2 s+1} \sum_{n=1}^{\infty} \sum_{a=1}^{2} \frac{\left[c_{n}\right]_{a} \gamma\left[s+n+\frac{1}{2}, v_{a}^{2}\right]}{\sqrt{4 \pi} v_{a}^{2\left(s+n+\frac{1}{2}\right)}}+\text { error } \quad .
$$

It is a remarkable fact that the pole arising in $\lim _{s \rightarrow-\frac{1}{2}} \Delta_{1} \varepsilon^{K(c)}(s)$ appears in the $n=0$ term of the asymptotic expansion. The residue takes a value such that this divergence is exactly cancelled by $\lim _{s \rightarrow-\frac{1}{2}} \Delta_{2} \varepsilon^{K(c)}(s)$. Explicit computation of (15) has been carried out for $\sigma=2$ and we have perfect concordance with the data depicted in the Figure 5 . In this case, the coefficients $c_{n}$ vanish, such that $\frac{\partial}{\partial c} \Delta \varepsilon^{K(c)}(s)=0$.

\section{Jacobi fields, kink instability and resonances}

In the $\mathcal{C}_{(2,2)}^{ \pm \mp}$ topological sectors, things are more difficult. As critical points of the energy functional, the topological defects must satisfy the Euler-Lagrange equations

$$
\frac{d^{2} \phi_{1}}{d x^{2}}=2 \phi_{1}\left(4 \phi_{1}^{2}+2 \sigma(1+\sigma) \phi_{2}^{2}-1\right) \quad \frac{d^{2} \phi_{2}}{d x^{2}}=\sigma \phi_{2}\left(4(\sigma+1) \phi_{1}^{2}+2 \sigma \phi_{2}^{2}-1\right) \quad .
$$

If $\sigma=2$ and $\sigma=\frac{1}{2}$, we are able to obtain all the kinks in these sectors of the model because the associated dynamical problem is integrable.

- For $\sigma=2$ in the $\mathcal{C}_{(2,2)}^{ \pm \mp}$ topological sectors, we find

$$
\vec{\phi}_{T K 2^{\prime}}[x ; b]=\frac{(-1)^{\alpha}}{2} \frac{\sqrt{b^{2}-1}}{\cosh 2(x+a)+b} \vec{e}_{1}+\frac{(-1)^{\beta}}{2} \frac{\sinh 2(x+a)}{\cosh 2(x+a)+b} \vec{e}_{2}
$$

- For $\sigma=\frac{1}{2}$, in the $\mathcal{C}_{(2,2)}^{ \pm \mp}$ topological sectors we have the family of solutions:

$$
\begin{aligned}
\vec{\phi}_{T K 2^{\prime}}[x ; b] & =(-1)^{\alpha} \frac{\sinh b \sinh (x+a)}{\cosh ^{2}(x+a)+2 \cosh b \cosh (x+a)+1} \vec{e}_{1}+ \\
& +(-1)^{\beta} \frac{-\sinh (x+a)}{\sqrt{\cosh ^{2}(x+a)+2 \cosh b \cosh (x+a)+1}} \vec{e}_{2}
\end{aligned}
$$


For generic $\sigma$, only the TK1' kinks are known explicitly in $\mathcal{C}_{(2,2)}^{ \pm \mp}$ :

$$
\vec{\phi}_{\mathrm{TK} 1^{\prime}}(x)=(-1)^{\alpha} \frac{1}{\sqrt{2 \sigma}} \tanh \sqrt{\frac{\sigma}{2}}(x+a) \quad \text {. }
$$

Defining $z=\sqrt{\frac{\sigma}{2}} x$, we write the Hessian for the TK1' kinks as

$$
\mathcal{J}=\frac{\sigma}{2}\left(\begin{array}{cc}
-\frac{d^{2}}{d z^{2}}+4-\frac{4(1+\sigma)}{\sigma} \operatorname{sech}^{2} z & 0 \\
0 & -\frac{d^{2}}{d z^{2}}+4-6 \operatorname{sech}^{2} z
\end{array}\right) .
$$

$\mathcal{J}$ is a diagonal matrix differential operator that includes as the $\mathcal{J}_{22}$ matrix element the very well known Posch-Teller Schrödinger operator, with $c_{0}^{2}=4$ and $u_{0}=6$. $\mathcal{J}_{11}$ is also of Posch-Teller type - $c_{0}^{2}=4, u_{0}=\frac{4(1+\sigma)}{\sigma}$-, and deserves a closer analysis. The eigenvalues $\omega_{n}=4-\left[\kappa-\left(n+\frac{1}{2}\right)\right]^{2}$, $\kappa=\sqrt{\frac{4(1+\sigma)}{\sigma}+\frac{1}{4}}, n=0,1,2, \cdots, I\left[\kappa-\frac{1}{2}\right]-1$ belong to the discrete spectrum of $\mathcal{J}_{11}$. Thus,

$$
\operatorname{Spec} \mathcal{J}=\cup_{n=0}^{I\left[\kappa-\frac{1}{2}\right]-1}\left\{4-\left[\kappa-\left(n+\frac{1}{2}\right)\right]^{2}\right\} \cup\left\{k_{1}^{2}+4\right\} \cup\{0\} \cup\{3\} \cup\left\{k_{2}^{2}+4\right\}_{k_{2} \in \mathbb{R}}
$$

The phase shifts for the continuous spectrum are

$$
\delta\left(k_{1}\right)=\delta_{+}\left(k_{1}\right)+\delta_{-}\left(k_{1}\right) ; \quad \delta\left(k_{2}\right)=-2 \arctan \frac{3 k_{2}}{2-k_{2}^{2}} \quad .
$$

The reflection coefficient associated to the Schrödinger operator $\mathcal{J}_{11}$ is not zero if $\sigma \neq \frac{4}{j(j+1)-4}$ with $j$ a positive integer number greater than or equal to 2 . Therefore, there are also in general even and odd phase shifts,

$$
\delta_{ \pm}\left(k_{1}\right)=\frac{1}{4} \arctan \left(\frac{\operatorname{Im}\left(T\left(k_{1}\right) \pm R\left(k_{1}\right)\right)}{\operatorname{Re}\left(T\left(k_{1}\right) \pm R\left(k_{1}\right)\right)}\right)
$$

to be read from the transmission and reflection

$$
T\left(k_{1}\right)=\frac{\Gamma\left(\frac{1}{2}+\kappa-i k_{1}\right) \Gamma\left(\frac{1}{2}-\kappa-i k_{1}\right)}{\Gamma\left(1-i k_{1}\right) \Gamma\left(-i k_{1}\right)} \quad, \quad R\left(k_{1}\right)=\frac{\Gamma\left(\frac{1}{2} \kappa-i k_{1}\right) \Gamma\left(\frac{1}{2}-\kappa^{2}-i k_{1}\right) \Gamma\left(i k_{1}\right)}{\Gamma(1+\kappa) \Gamma\left(1-\kappa^{2}\right) \Gamma\left(-i k_{1}\right)}
$$

coefficients.

The physical and geometrical information encoded in the spectrum of $\mathcal{J}$ is as follows:

- There are two bound states in the tangent direction to the $T K 1^{\prime} \operatorname{kink}$ in $\mathcal{C}_{(2 ; 2)}^{ \pm \mp}$. The first of them is the translational zero mode and the second one is interpreted as usual: a scalar boson "polarized" in internal space in the $\vec{e}_{2}$ direction is captured by the kink. The scattering eigenstates correspond to $\vec{e}_{1}$-polarized scalar bosons moving in the kink background.

- There are $I\left[\kappa-\frac{1}{2}\right]-1$ bound states in the orthogonal direction to the TK1 $1^{\prime}$ kink in $\mathcal{C}_{(2 ; 2)}^{ \pm \mp}$. The associated eigenvalues are positive if $\sigma>2$ and thus the TK1' kink is stable in this regime. 
- When $\sigma<2$, there are some negative eigenvalues in $\operatorname{Spec}(\mathcal{J})$ and the $\mathrm{TK} 1^{\prime}$ kink is unstable against the associated fluctuations. The number of negative eigenvalues and the degree of instability increase with decreasing $\sigma$ : if $\sigma \in\left(\frac{4}{j(j-1)-4}, \frac{4}{j(j+1)-4}\right]$ the spectrum of $\mathcal{J}_{11}$ contains $j-2$ negative eigenvalues and there exist $j-2$ instability directions around the TK1 ${ }^{\prime}$ kink in the $\mathcal{C}_{(2 ; 2)}^{ \pm \mp}$ sectors. For an analysis of kink stability in multi-component scalar field theory, see [33].

- At the critical values $\sigma=\frac{4}{j(j+1)-4}$ there is a jump in the number of negative eigenvalues and the Jacobi field

$$
\psi_{J}(q) \cong\left(1-q^{2}\right)_{2} F_{1}\left[\frac{5}{2}+\sqrt{j(j+1)+\frac{1}{4}}, \frac{5}{2}-\sqrt{j(j+1)+\frac{1}{4}}, 3, \frac{1}{2}(1+q)\right] \quad ; \quad q=\tanh z
$$

becomes a bound state belonging to the discrete spectrum of $\mathcal{J}_{11}$. The Morse index theorem, [33], tells us that there is a continuous family of TK2' kinks in the $\mathcal{C}_{(2 ; 2)}^{ \pm \mp}$ sectors if $\sigma=\frac{4}{j(j+1)-4}$. The members of this family are the flow lines of the gradient of a second superpotential $\tilde{W}$ that has two critical points at $\vec{\phi}_{V_{2}^{ \pm}}= \pm \frac{\sqrt{j(j+1)-4}}{2 \sqrt{2}} \vec{e}_{2}$ and $j-2$ branching points: the zeroes of $\psi_{J}(x) \vec{e}_{2}$, see [33] .
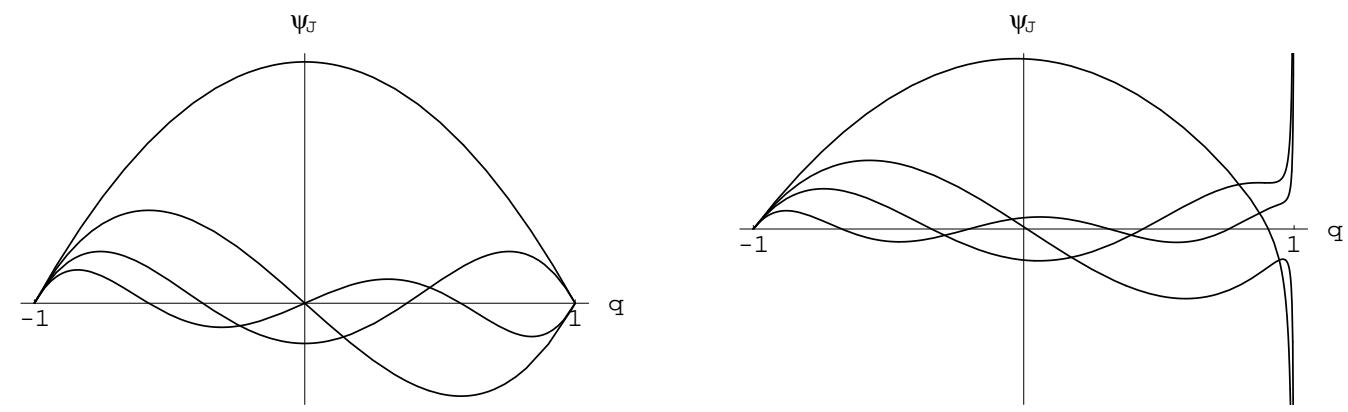

Figure 6: Graphics of $\psi_{J}(q)$ for $j=2, j=3, j=4, j=5$ (left), for non-critical values of $\sigma$ close to the critical ones (right).

Therefore, the TK1' kink gives rise to a bona fide eigenstate of the Hamiltonian only if $\sigma>2$. Here we shall not give $\Delta M\left(\mathrm{TK}^{\prime}\right)$, because the computation is identical to that developed for $\Delta M$ (TK1) if $\sigma \geq 2$ but gives rise to a complex mass if $\sigma^{2}<2$; the TK1' kink state should be interpreted as a resonance in this regime.

\section{Appendix: mode number cutoff regularization method}

In this Appendix we present a derivation of the basic equation (6) in Section $\S 3$. Given the $\mathcal{K}$ and $\mathcal{V}$ Schrodinger operators

$$
\mathcal{V}=-\frac{d^{2}}{d x^{2}}+v^{2} \quad, \quad \mathcal{K}=-\frac{d^{2}}{d x^{2}}+v^{2}-V(x),
$$


respectively ruling the small fluctuations around the vacuum and the kink, the renormalized zero point energy is:

$$
\Delta_{1} \varepsilon^{\mathrm{K}}=\frac{\hbar m}{2}\left(\sum_{\text {Spec } \mathcal{K}} \omega_{n}-\sum_{\text {Spec } \mathcal{V}} \bar{\omega}_{n}\right) \quad .
$$

Here, $v^{2}=\left.\frac{d^{2} U}{d \psi^{2}}\right|_{\psi_{\mathrm{V}}}$ is a constant, and $v^{2}-V(x)=\left.\frac{d^{2} U}{d \psi^{2}}\right|_{\psi_{\mathrm{K}}}$ gives rise to a potential well $V(x)$, which rapidly decreases to its asymptotic value: $\lim _{x \rightarrow \pm \infty} V(x)=0$; note that, as in the main text, we use dimensionless eigenvalues and recover the dimension in the final response through the overall constant of (18). The spectrum of both $\mathcal{K}$ and $\mathcal{V}$ is continuous and some regularization is needed to compute the infinite sums in the Casimir energy (18). Over the last few years it became clear that the correct regularization procedure is based on a cut-off in the number of fluctuation modes, a number that must be taken to be "equal" in the vacuum and kink sectors, see [12]. More recently, controversy arose about whether an energy cut-off regularization can yield the correct answer, see [34] and [35]. This is a subtle matter indeed, but all the evidence leads us to rely on the mode number regularization method.

Before deriving (6), we outline the set-up pointed out in [14] that underlies our approach below. The problem is discretised by confining the system to a box of length $2 L$, which is much greater than any length scale characteristic of the potential term or of any of its bound states, and the limit $L \rightarrow \infty$ is taken at the end of the analysis. In order to specify the boundary conditions we distinguish between (a) eigenstates with eigenvalue greater than $v^{2}$, giving rise to the continuous part of the spectrum, and (b) those with eigenvalue lower than or equal to $v^{2}$, corresponding respectively to bound or half-bound states. Fictitious boundary conditions $\psi( \pm L)=0$ are imposed on the eigenstates of type (a), but we can safely anticipate the limit $L \rightarrow \infty$ for states of type (b) and use for them the eigenfunctions of the actual bound or half-bound states respectively decrease exponentially or go to a finite constant at long distances. In any case, we stress the fact that the effects induced by the boundary conditions disappear when the $L \rightarrow \infty$ limit is taken. Nevertheless, we shall approach first the problem by focusing on operators $\mathcal{K}$ without half-bound states. This particular case (tackling operators which involve potentials without reflection, such as those in the kink and soliton of the well known $\phi^{4}$ and Sine-Gordon model) will be dealt with later.

Apart from restricting the spectra of $\mathcal{V}$ and $\mathcal{K}$

$$
\mathcal{V} \psi[k ; x]=\bar{\omega}^{2}(k) \psi[k ; x] \quad, \quad \mathcal{K} \psi[q ; x]=\omega^{2}(q) \psi[q ; x]
$$

to those eigenfunctions of type (a) satisfying Dirichlet boundary conditions $\psi( \pm L)=0$, we shall only consider symmetric potentials $V(x)=V(-x)$ which allows us the choice of odd, $\psi(x)=$ $-\psi(-x)$, and, even, $\psi(x)=\psi(-x)$, eigenstates complying with the boundary conditions. This breaks the degeneracy that arises by imposing periodic conditions on eigenfunctions with non defined-parity (plane waves), and hence skips some problems arising when the number of bound states is odd.

A brief description of the eigenvalues and eigenfunctions of $\mathcal{V}$ and $\mathcal{K}$ in a finite box follows.

1. Spectrum of $\mathcal{V}$

- Half-bound state. 
The constant function,

$$
\psi_{0}(x)=\text { constant } \quad,
$$

is an eigenfunction of $\mathcal{V}$ with eigenvalue $v^{2}$ that do not grow at $x= \pm \infty$.

- Odd eigenfunctions

If $n^{-}$is a natural number strictly positive and the wave vectors $k_{n^{-}}$satisfy

$$
k_{n^{-}} \cdot L=\pi n^{-} \quad, \quad n^{-} \in \mathbb{N}^{+} \quad,
$$

then the odd functions, $\psi_{n^{-}}(x)=-\psi_{n^{-}}(-x)$,

$$
\psi_{n^{-}}(x)=A_{n^{-}} \cdot \sin \left(k_{n^{-}} \cdot x\right)
$$

belong to the spectrum of $\mathcal{V}$ with eigenvalue $\bar{\omega}^{2}\left(k_{n^{-}}\right)=k_{n^{-}}^{2}+v^{2}$. Since $\left(k_{n^{-}+1}-k_{n^{-}}\right) L=$ $\pi$ the density of odd eigenstates is: $\bar{\rho}^{-}(k)=\frac{L}{\pi}$.

- Even eigenfunctions

If $n^{+}$is a natural number and the wave vectors $k_{n^{+}}$satisfy

$$
k_{n^{+}} \cdot L=\pi\left(n^{+}+\frac{1}{2}\right) \quad, \quad n^{+} \in \mathbb{N} \quad,
$$

then the even functions, $\psi_{n^{+}}(x)=\psi_{n^{+}}(-x)$,

$$
\psi_{n^{+}}(x)=A_{n^{+}} \cdot \cos \left(k_{n^{+}} \cdot x\right)
$$

belong to the spectrum of $\mathcal{V}$ with eigenvalue $\bar{\omega}^{2}\left(k_{n^{+}}\right)=k_{n^{+}}^{2}+v^{2}$. Now, $\bar{\rho}^{+}(k)=\frac{L}{\pi}$.

\section{Spectrum of $\mathcal{K}$}

- Bound states:

We assume that there exist $l$ bound states with $\omega^{2} \leq v^{2}$. In particular we denote by $l^{+}$the number of symmetric bound states and by $l^{-}$the number of antisymmetric states, $l=l^{+}+l^{-}$. If $\omega_{l}^{2}=v^{2}$, the highest bound state becomes a half-bound one and contributes with a weight of $s_{l}=\frac{1}{2}$ to the mass quantum correction.

- Odd eigenfunctions.

There are odd eigenfunctions which asymptotically are of the form:

$$
\psi_{n^{-}}(x) \stackrel{x \rightarrow L}{\simeq} A_{n^{-}} \cdot \sin \left[q_{n^{-}} \cdot x+\delta^{-}\left(q_{n^{-}}\right)\right] .
$$

The odd phase shifts $\delta^{-}\left(q_{n^{-}}\right)$are defined in terms of the transmission and reflection coefficients, see Section $\S 3$, but now the boundary conditions require:

$$
q_{n^{-}} \cdot L+\delta^{-}\left(q_{n^{-}}\right)=\pi n^{-} \quad, \quad n^{-} \in \mathbb{N} \quad .
$$

Unitarity, transparency at $q=\infty$, and, continuity of the wave function at $q=0$ (threshold) allow us to set the phase ambiguity as in [14]:

$$
\lim _{q \rightarrow \infty} \delta^{-}(q)=0 \quad, \quad \lim _{q \rightarrow 0^{+}} \delta^{-}(q)=\pi l^{-} \quad .
$$


The question arises: what is the minimum value $n^{-}=n_{0}^{-}$? For very large $L, q_{n_{0}^{-}}$is very small and (19) becomes:

$$
q_{n_{0}^{-}} \cdot\left(L+\delta^{-}\left(0^{+}\right)\right)+\delta^{-}\left(0^{+}\right)=\pi n_{0}^{-} \quad .
$$

Here, $\delta^{\prime-}\left(0^{+}\right)$is the limit of the left-hand derivative of the antisymmetric phase shift when $q$ goes to zero from the right. The minimum value of $n^{-}=n_{0}^{-}$is therefore $n_{0}^{-}=l^{-}$. Since $\left(q_{n^{-}+1}-q_{n^{-}}\right) L+\delta^{-}\left(q_{n^{-}+1}\right)-\delta\left(q_{n^{-}}\right)=\pi$ the density of antisymmetric states is:

$$
\rho^{-}(q)=\frac{L}{\pi}+\frac{1}{\pi} \frac{\partial \delta^{-}(k)}{\partial k}
$$

- Even eigenfunctions.

There are even eigenfunctions which asymptotically are of the form:

$$
\psi_{n^{+}}(x) \stackrel{x \rightarrow L}{\simeq} A_{n^{+}} \cdot \cos \left[q_{n^{+}} \cdot x+\delta^{+}\left(q_{n^{+}}\right)\right] \quad .
$$

The phase shifts $\delta^{+}\left(q_{n^{+}}\right)$are defined in terms of the transmission and reflection coefficients, see Section $\S 3$, and the boundary conditions require:

$$
q_{n^{+}} \cdot L+\delta^{+}\left(q_{n^{+}}\right)=\pi\left(n^{+}+\frac{1}{2}\right) \quad, \quad n^{+} \in \mathbb{N} \quad .
$$

Again we set the phase ambiguity by invoking Levinson's theorem as in [14]:

$$
\lim _{q \rightarrow \infty} \delta^{+}(q)=0 \quad, \quad \lim _{q \rightarrow 0^{+}} \delta^{+}(q)=\pi\left(l^{+}-\frac{1}{2}\right)
$$

where $l^{+}$is the number of even bound states.

From

$$
q_{n_{0}^{+}} \cdot\left(L+\delta^{\prime+}\left(0^{+}\right)\right)+\delta^{+}\left(0^{+}\right)=\pi\left(n_{0}^{+}+\frac{1}{2}\right)
$$

we read the minimum possible value of $q_{n_{0}^{+}}: n_{0}^{+}=l^{+}$. Now,

$$
\rho^{+}(q)=\frac{L}{\pi}+\frac{1}{\pi} \frac{\partial \delta^{+}(k)}{\partial k}
$$

We stress that due to the parity of the eigenfunctions there is no need to consider negative values of $n^{ \pm}$.

The regularized version of formula (18) reads:

$$
\begin{aligned}
\Delta_{1} \varepsilon^{K} & =\frac{\hbar m}{2} \sum_{i=1}^{l} \omega_{i}+ \\
& +\frac{\hbar m}{2}\left[\sum_{n^{+}=l^{+}}^{N} \sqrt{q_{n^{+}}^{2}+v^{2}}-\sum_{n^{+}=0}^{N_{0}} \sqrt{k_{n^{+}}^{2}+v^{2}}\right]+ \\
& +\frac{\hbar m}{2}\left[\sum_{n^{-}=l^{-}}^{N^{\prime}} \sqrt{q_{n^{-}}^{2}+v^{2}}-\sum_{n^{-}=0}^{N_{0}^{\prime}} \sqrt{k_{n^{-}}^{2}+v^{2}}\right]
\end{aligned}
$$


The first row collects the contributions of the $l=l^{+}+l^{-}$bound states of $\mathcal{K}$. In the other two rows, the contributions of even and odd eigenfunctions in the continuous spectra are accounted for. Note that we have added the contribution of the constant mode of $\mathcal{V}$ to the odd sector because it corresponds to the lower eigenvalue of $\mathcal{V}$. We stick to mode number cut-off regularization, i.e. we impose $N=N_{0}$ and $N^{\prime}=N_{0}^{\prime}$. This choice balances the number of states of $\mathcal{K}$ and $\mathcal{V}$ involved in the above formula. We have distinguished the number of symmetric and antisymmetric states, $N$ and $N^{\prime}$, although we shall consider either $N^{\prime}=N+1$ or $N^{\prime}=N$.

To measure the contribution of $\mathcal{K}$ with respect to the contribution of $\mathcal{V}$ to the Casimir energy mode by mode, we write the second row in the form:

$$
\begin{aligned}
& \frac{\hbar m}{2}\left[\sum_{n^{+}=0}^{N}\left(\sqrt{q_{n^{+}}^{2}+v^{2}}-\sqrt{k_{n^{+}}^{2}+v^{2}}\right)-\sum_{n^{+}=0}^{l^{+}-1} \sqrt{q_{n^{+}}^{2}+v^{2}}\right] \\
\simeq & \frac{\hbar m}{2}\left[-\sum_{n^{+}=0}^{N} \frac{k_{n^{+}} \delta^{+}\left(k_{n^{+}}\right)}{L \sqrt{k_{n^{+}}^{2}+v^{2}}}-\sum_{n^{+}=0}^{l^{+}-1} \sqrt{q_{n^{+}}^{2}+v^{2}}+\theta\left(\frac{1}{L^{2}}\right)\right] \\
\stackrel{\substack{L \rightarrow \infty \\
\text { 壬 }}}{ }- & -\frac{\hbar m}{2}\left[\int_{0}^{\infty} \frac{d k}{\pi} \frac{d \omega(k)}{d k} \delta^{+}(k)+l^{+} v\right] .
\end{aligned}
$$

A similar process for the odd eigenfunctions gives:

$$
\begin{aligned}
& \frac{\hbar m}{2}\left[\sum_{n^{-}=0}^{N^{\prime}}\left(\sqrt{q_{n^{-}}^{2}+v^{2}}-\sqrt{k_{n^{-}}^{2}+v^{2}}\right)-\sum_{n^{-}=0}^{l^{-}-1} \sqrt{q_{n^{-}}^{2}+v^{2}}\right] \\
& \simeq \frac{\hbar m}{2}\left[-\sum_{n^{-}=0}^{N^{\prime}} \frac{k_{n^{-}} \delta^{-}\left(k_{n^{-}}\right)}{L \sqrt{k_{n^{-}}^{2}+v^{2}}}-\sum_{n^{-}=0}^{l^{-}-1} \sqrt{q_{n^{-}}^{2}+v^{2}}+\theta\left(\frac{1}{L^{2}}\right)\right] \\
& \stackrel{\substack{L \rightarrow \infty \\
N^{\prime} \rightarrow \infty}}{\simeq}-\frac{\hbar m}{2}\left[\int_{0}^{\infty} \frac{d k}{\pi} \frac{d \omega(k)}{d k} \delta^{-}(k)+l^{-} v\right] .
\end{aligned}
$$

The sum of all contributions plus a partial integration,

$$
\begin{aligned}
\Delta_{1} \varepsilon^{\mathrm{K}}= & \frac{\hbar m}{2} \sum_{i=1}^{l} \omega_{i}- \\
& -\frac{\hbar m}{2} l^{+} v-\left.\frac{\hbar m}{2 \pi} \delta^{+}(k) \omega(k)\right|_{0} ^{\infty}+\frac{\hbar m}{2 \pi} \int_{0}^{\infty} d k \frac{d \delta^{+}(k)}{d k} \omega(k) \\
& -\frac{\hbar m}{2} l^{-} v-\left.\frac{\hbar m}{2 \pi} \delta^{-}(k) \omega(k)\right|_{0} ^{\infty}+\frac{\hbar m}{2 \pi} \int_{0}^{\infty} d k \frac{d \delta^{-}(k)}{d k} \omega(k),
\end{aligned}
$$

and the asymptotic behaviour of the total phase shift,

$$
\delta^{+}(k) \stackrel{k \rightarrow \infty}{\cong} \frac{1}{k} \int_{0}^{\infty} d x V(x) \cos ^{2}(k x) \quad \delta^{-}(k) \stackrel{k \rightarrow \infty}{\cong} \frac{1}{k} \int_{0}^{\infty} d x V(x) \sin ^{2}(k x)
$$

show that:

$$
\begin{aligned}
\Delta_{1} \varepsilon^{\mathrm{K}}= & \frac{\hbar m}{2} \sum_{i=1}^{l} \omega_{i}-\frac{\hbar m}{2} l^{+} v+\frac{\hbar m}{2 \pi} \delta^{+}(0) v-\frac{\hbar m}{2} l^{-} v+\frac{\hbar m}{2 \pi} \delta^{-}(0) v- \\
& -\frac{\hbar m}{2 \pi} \int_{0}^{\infty} d x V(x)\left[\cos ^{2}(k x)+\sin ^{2}(k x)\right]+\frac{\hbar m}{2 \pi} \int_{0}^{\infty} d k \frac{d\left[\delta^{+}(k)+\delta^{-}(k)\right]}{d k} \omega(k)
\end{aligned}
$$


From the 1D Levinson theorem we notice that the two last terms in the first line of the above formula cancel each other but the two preceding ones leave a contribution: $-\frac{v}{2}$. Therefore, the formula for the kink Casimir energy reads:

$$
\Delta_{1} \varepsilon^{\mathrm{K}}=\frac{\hbar m}{2}\left[\sum_{i=1}^{l} \omega_{i}-\frac{v}{2}+\frac{1}{\pi} \int_{0}^{\infty} d k \frac{d \delta(k)}{d k} \omega(k)-\frac{\langle V(x)\rangle}{2 \pi}\right],
$$

where $\langle V(x)\rangle=\int_{-\infty}^{\infty} V(x) d x=2 \int_{0}^{\infty} V(x) d x$ is the expectation value of the potential term and the contribution of the continuous spectrum is encoded in the total phase shift: $\delta(k)=\delta^{+}(k)+\delta^{-}(k)$. We call attention to the contribution $-\frac{\hbar m v}{4}$, which tells us that the contribution of the mode with $k=0$ wave vector in $\operatorname{Spec} \mathcal{V}$ is half the contribution of a bona fide bound state -hence, the name-.

The generalization of this approach to tackle the presence of a bound state with $k=0$ and $\omega^{2}=v^{2}$ in SpecK (half-bound state) is direct. The Levinson theorem,

$$
\delta_{-}\left(0^{+}\right)=l_{-} \pi \quad, \quad \delta_{+}\left(0^{+}\right)=l_{+} \pi-\frac{\pi}{2} \quad
$$

works as above, but there is now a half-bound state that contributes with $\frac{1}{2}$ to both the number $l=l_{-}+l_{+}$and the mass quantum correction $\Delta_{1} \varepsilon^{K}$, see [13]. Thus, we write $(23)$ as $\delta\left(0^{+}\right)=$ $l \pi-\frac{\pi}{2}=n_{b} \pi$, and, bearing in mind the previous observation, the above calculations reproduce the results obtained in Reference [12]. The tricky point to realize is how the contribution of the threshold state turns $(23)$ into $\delta\left(q^{+}\right)=n_{b} \pi$, with $n_{b}$ the number of bound states with $\omega^{2}<v^{2}$. In the kink mass formula, the contribution of the half-state would cancel out the term $-\frac{v}{2}$, and everything is okay. For this reason, the formula shown in [12] is valid only for operators involving potentials without reflection. A final expression involving both of these cases provides the first row of formula (6)

$$
\Delta_{1} \varepsilon^{\mathrm{K}}=\frac{\hbar m}{2}\left[\sum_{i=1}^{l-1} \omega_{i}+s_{l} \omega_{l}-\frac{v}{2}+\frac{1}{\pi} \int_{0}^{\infty} d k \frac{d \delta(k)}{d k} \omega(k)-\frac{\langle V(x)\rangle}{2 \pi}\right],
$$

where $s_{l}=\frac{1}{2}$ if we deal with a reflectionless potential but $s_{l}=1$ if the reflection coefficient is non-zero.

\section{Acknowledgements}

E-mail correspondence with A. Rebhan, P. van Niewenhuizen and R. Wimmer about important points of their work on kink quantization is gratefully acknowledged. We also thank J.J. BlancoPillado for critical reading of the manuscript.

A.A.I. thanks to the Secretaria de Estado de Educación y Universidades of Spain for financial support. The work of W.G.F. has been partially supported by Oviedo University, Vicerrectorado de Investigacion grantMB-03-514-1.

\section{References}

[1] D. Olive and E. Witten, Phys. Lett. B78 (1978) 97; N. Seiberg and E. Witten, Nucl. Phys. B246 (1994) 19. 
[2] M. Duff, R. Khuri and J. Lu, Phys. Rep. 259 (1995) 213.

[3] G. Dvali and M. Shifman, Nucl. Phys. B504 (1997) 127; G. Gibbons and P. Townsend, Phys. Rev. Lett. 83 (1999) 172.

[4] R. Dashen, B. Hasslacher and A. Neveu, Phys. Rev. D10 (1974) 4130; Phys. Rev. D12 (1975) 3424.

[5] L. D. Faddeev and V. E. Korepin, Phys. Rep. 42C (1978) 1-87.

[6] A. D'Adda, R. Horsley and P. Di Vecchia, Phys. Lett. B76 (1978) 298; J. Schonfeld, Nucl. Phys. B161 (1979) 125; A. D'Adda, R. Horslet and P. di Vecchia, Phys. Lett. $76 B$ (1978) 4130 and Phys. Rev. D12 (1975) 3424; R. Horsley, Nucl. Phys. B151 (1979) 399.

[7] S. Rouhani, Nucl. Phys. B182 (1981) 462; H. Yamagishi, Phys. Lett. 147B (1984) 425; A.K. Chatterjee and P. Majumdar, Phys. Rev. D30 (1984) 844; Phys. Lett. 159B (1985) 37.

[8] A. Uchiyama, Nucl. Phys. B244 (1984) 57; Progr. Theor. Phys. 75 (1986) 1214; Nucl. Phys. B278 (1986) 121.

[9] R. K. Kaul and R. Rajaraman, Phys. Lett. B131 (1983) 357; J.F. Schonfeld, Nucl. Phys. B161 (1979) 125.

[10] C. Imbimbo and S. Mukhi, Nucl. Phys. B247 (1984) 471.

[11] M. Shifman, A. Vainshtein and M. Voloshin, Phys. Rev. D59 (1999) 45016.

[12] A. Rebhan and P. van Nieuwenhuizen, Nucl. Phys. B508 (1997) 449-467; H. Nastase, M. Stephanov, A. Rebhan and P. van Nieuwenhuizen, Nucl. Phys. B542 (1999) 471-514.

[13] N. Graham and R. Jaffe, Nucl. Phys. B544 (1999) 432 and Nucl. Phys. B549 (1999) 516.

[14] G. Barton, J. Phys. A18 (1985)479.

[15] M. Bordag, A. Goldhaber, P. van Nieuwenhuizen and D. Vassilevich, Phys. Rev. D66 (2002) 125014.

[16] A. Alonso-Izquierdo, W. Garcia Fuertes, M.A. Gonzalez Leon and J. Mateos Guilarte, Nucl. Phys. B635 (2002) 525-557.

[17] P. Gilkey, Invariance theory, the heat equation and the Atiyah-Singer index theorem, Publish or Perish, Delaware, 1984.

[18] A. Alonso-Izquierdo, W. Garcia Fuertes, M.A. Gonzalez Leon and J. Mateos Guilarte, Nucl. Phys. B638 (2002) 378-404.

[19] C. Montonen, Nucl. Phys. B112 (1976) 349; S. Sarker, S.E. Trullinger and A. R. Bishop, Phys. Lett. 59A (1976) 255-258.

[20] A. Goldhaber, A. Litvintsev and P. van Nieuwenhuizen, Phys. Rev. D67 (2003)105021; R. Wimmer, Quantization of supersymmetric solitons, hep-th/0109119. 
[21] A. Rebhan, P. van Nieuwenhuizen and R. Wimmer, New J. Phys. 4 (2002)31.

[22] D. Bazeia, J.R.S. Nascimento, R. Ribeiro, and, D. J. Toledo, J. Phys. A30 (1997) 8157-8166;

D. Bazeia, M.J. Dos Santos and R. F. Ribeiro, Phys. Lett. A208 (1995) 84-88.

[23] M. Shifman and M. Voloshin, Phys. Rev. D57 (1998) 2590.

[24] A. Alonso Izquierdo, M.A. Gonzalez Leon and J. Mateos Guilarte, Phys. Rev. D65 (2002)085012.

[25] G. S. Dias, E. L. Graca and R. de Lima Rodrigues, Stability equation and two-component eigenmode for domain walls in a scalar potential model, hep-th/0205195.

[26] M. Eto and N. Sakai, Solvable models of domain walls in $\mathcal{N}=1$ supergravity, hep-th/0307276.

[27] A. Alonso-Izquierdo, M. A. Gonzalez Leon, J. Mateos Guilarte and M. de la Torre Mayado, Phys. Rev. D66 (2002) 105022.

[28] P. G. Drazin and R. S. Johnson, Solitons: an introduction, Cambridge University Press, Cambridge, 1996.

[29] P.M. Morse and H. Feshbach, Methods of Theoretical Physics, McGraw-Hill Book Company, Inc., New York-Toronto-London, 1953.

[30] M. Abramowitz and I. Stegun, Handbook of Mathematical Functions with Formulas, Graphs and Mathematical Tables, Dover, New York, 1992.

[31] K. Fujikawa and P. van Nieuwenhuizen, Topological anomalies from the path integral measure in superspace, hep-th/0305144.

[32] I. Avramidi and R. Schimming, J. Math. Phys. 36 (1995) 5042.

[33] A. Alonso Izquierdo, M.A. Gonzalez Leon and J. Mateos Guilarte, Nonlinearity 15 (2002) 1097-1125.

[34] G. Flores-Hidalgo, Phys. Lett. B542 (2002) 282.

[35] A. Rebhan, P. van Nieuwenhuizen, and, R. Wimmer, Phys. Lett. B552 (2003) 17. 